\title{
A Unitary Extension of Virtual Permutations
}

\author{
Paul Bourgade $^{1}$, Joseph Najnudel ${ }^{2}$, and Ashkan Nikeghbali ${ }^{2}$ \\ ${ }^{1}$ Department of Mathematics, Harvard University, 1 Oxford street, \\ Cambridge, MA 02138, USA and ${ }^{2}$ Institut für Mathematik, Universität \\ Zürich, Winterthurerstrasse 190, 8057 Zürich, Switzerland
}

Correspondence to be sent to: ashkan.nikeghbali@math.uzh.ch

Analogously to the space of virtual permutations [5], we define projective limits of isometries: these sequences of unitary operators are natural in the sense that they minimize the rank norm between successive matrices of increasing sizes. The space of virtual isometries we construct this way may be viewed as a natural extension of the space of virtual permutations of Kerov et al. [5] as well as an extension of the space of virtual isometries of Neretin [9]. We then derive with purely probabilistic methods an almost sure convergence for these random matrices under the Haar measure: for a coherent Haar measure on virtual isometries, the smallest normalized eigenangles converge almost surely to a point process whose correlation function is given by the sine kernel. This almost sure convergence actually holds for a larger class of measures as is proved by Borodin and Olshanski [1]. We give a different proof, probabilistic in the sense that it makes use of martingale arguments and shows how the eigenangles interlace when going from dimension $n$ to $n+1$. Our method also proves that for some universal constant $\varepsilon>0$, the rate of convergence is almost surely dominated by $n^{-\varepsilon}$ when the dimension $n$ goes to infinity. 


\section{Introduction}

In [5], the virtual permutations have been introduced in order to study some asymptotic properties of the symmetric group $\mathcal{S}_{n}$ of order $n \in \mathbb{N}$, when $n$ goes to infinity. The space $\mathcal{S}^{\infty}$ of virtual permutation can be defined as follows. For $n \geq m \geq 1, \pi_{n, m}$ denotes the application from $\mathcal{S}_{n}$ to $\mathcal{S}_{m}$ such that for $\sigma \in \mathcal{S}_{n}, \pi_{n, m}(\sigma) \in \mathcal{S}_{m}$ is obtained from $\sigma$ by deleting all the elements of $\{m+1, \ldots, n\}$ from its cycle structure; then $\mathcal{S}^{\infty}$ is the projective limit of $\left(\mathcal{S}_{n}\right)_{n \geq 1}$, that is, the set of sequences $\left(\sigma_{n}\right)_{n \geq 1}$ of permutations such that for $n \geq 1$, $\sigma_{n} \in \mathcal{S}_{n}$ and for $n \geq m \geq 1, \sigma_{m}=\pi_{n, m}\left(\sigma_{n}\right)$. A virtual permutation $\left(\sigma_{n}\right)_{n \geq 1}$ can naturally be constructed by the so-called Chinese restaurant process (see, e.g., [13]), as follows:

(1) $\sigma_{1}$ is the unique permutation in $\mathcal{S}_{1}$;

(2) for $n \geq 1, \sigma_{n+1}$ is obtained from $\sigma_{n}$ either by adding $n+1$ as a fixed point, or by inserting $n+1$ inside a cycle of $\sigma_{n}$.

If the space $\mathcal{S}^{\infty}$ is endowed with the $\sigma$-algebra generated by the coordinates $\left(\sigma_{n}\right)_{n \geq 1}$, and if $\left(\mu_{n}\right)_{n \geq 1}$ is a sequence of probability measures, $\mu_{n}$ defined on $\mathcal{S}_{n}$, such that for all $n \geq 1$, $\mu_{n}$ is the image of $\mu_{n+1}$ by $\pi_{n+1, n}$, then there exists a unique probability measure on $\mathcal{S}^{\infty}$ such that its $n$th projection is equal to $\mu_{n}$ for all $n \geq 1$ (this result can be deduced from the Carathéodory theorem on extensions of measures). Among all the probability measures on $\mathcal{S}^{\infty}$, those which are invariant by conjugation (i.e., for all $n \geq 1$, their projection on $\mathcal{S}_{n}$ is invariant by conjugation with any element of $\mathcal{S}_{n}$ ) are called central measures and they have been studied in detail by Tsilevich (see $[15,16])$. The main result is the following: there exists a family $\left(\mu^{X}\right)_{X \in \Sigma}$ of particular central measures (called ergodic measures), indexed by the set

$$
\Sigma=\left\{x=\left(x_{1}, x_{2}, \ldots, x_{k}, \ldots\right), x_{1} \geq x_{2} \geq \cdots \geq x_{k} \geq \cdots \geq 0, x_{1}+x_{2}+\cdots+x_{k}+\cdots \leq 1\right\}
$$

such that every central measure $\mu$ can be written as follows:

$$
\mu=\int_{\Sigma} \mu^{X} \mathrm{~d} v(x),
$$

where $v$ is a probability measure on $\Sigma$. Now, if $x \in \Sigma$ is fixed, and if $\left(\sigma_{n}\right)_{n \geq 1}$ is a virtual permutation following the ergodic probability distribution $\mu^{x}$, then its global cycle structure determines a random partition of the set of positive integers, and by the general results of Kingman (see [6-8]), the following properties hold:

(1) The sets of the partition are either singletons (corresponding to fixed points of permutations) or have a strictly positive asymptotic density. 
(2) For all $k \geq 1$, the $k$ th largest cycle length of $\sigma_{n}$ (zero if $\sigma_{n}$ has less than $k$ cycles), divided by $n$, tends almost surely to $x_{k}$ when $n$ goes to infinity.

This property of convergence of the cycle lengths can easily be translated into an almost sure convergence of the point process of the eigenangles of the corresponding permutation matrices, if the angles associated to the permutation $\sigma_{n}$ are multiplied by a factor $n$. In this way, one obtains a deterministic limiting point process, containing the multiples of $2 \pi / x_{k}$ for all $k \geq 1$. This property of almost sure convergence can be extended to the general case of central measures: in this case, the limiting point process is random, and the sequence $\left(x_{k}\right)_{k \geq 1}$ of asymptotic cycle lengths follows the distribution $v$ defined by (1.1). An interesting family of central measures is obtained by taking, for a given parameter $\theta \in \mathbb{R}_{+}^{*}, v$ equal to the law of a Poisson-Dirichlet process of parameter $\theta$. In this case, $\mu$ is the so-called Ewens measure of parameter $\theta$, that is the unique measure under which for all $n \geq 1$, the coordinate $\sigma_{n}$ satisfies the following: for all $\sigma \in \mathcal{S}_{n}$,

$$
\mathbb{P}_{\mu}\left[\sigma_{n}=\sigma\right]=\frac{\theta^{k_{\sigma}}}{\theta(\theta+1) \cdots(\theta+n-1)},
$$

where $k_{\sigma}$ denotes the number of cycles of $\sigma$. In particular, for $\theta=1, \mu$ is the Haar measure on $\mathcal{S}^{\infty}$, that is the unique measure such that for all $n \geq 1$, the $n$th projection $\sigma_{n}$ follows the uniform measure on $\mathcal{S}_{n}$ under $\mu$. A random virtual permutation following the Ewens measure of parameter $\theta$ can be constructed by the Chinese restaurant process in a convenient way: conditionally on $\sigma_{n}, n+1$ is a fixed point of $\sigma_{n+1}$ with probability $\theta /(\theta+n)$, otherwise, it is inserted inside the cycle structure of $\sigma_{n}$, each of the $n$ possible places having the same probability $1 /(\theta+n)$.

A similar study has been made by Olshanski and Vershik [10], for the space $\mathcal{H}$ of the infinite-dimensional hermitian matrices, that is, the families $\left(M_{i j}\right)_{i, j \geq 1}$ of complex numbers such that $M_{i j}=\bar{M}_{j i}$ for all $i, j \geq 1$. A central measure on $\mathcal{H}$, endowed with the $\sigma$ algebra generated by the coordinates $M_{i j}, i, j \geq 1$, is defined as a probability measure $\mu$ such that for all $n \geq 1$, the image of $\mu$ by the projection $\left(M_{i j}\right)_{i, j \geq 1} \mapsto\left(M_{i j}\right)_{1 \leq i, j \leq n}$ from $\mathcal{H}$ to the space of $n \times n$ hermitian matrices is invariant by conjugation with any $n \times n$ unitary matrix. In [10], it is proved that there exists a family $\left(\mu^{X}\right)_{x \in \Delta}$ of particular measures, again called ergodic measures, indexed by the set

$$
\begin{aligned}
\Delta= & \left\{x=\left(\gamma_{1}, \gamma_{2}, x_{1}^{+}, x_{2}^{+}, \ldots, x_{k}^{+}, \ldots, x_{1}^{-}, x_{2}^{-}, \ldots, x_{k}^{-}, \ldots\right) ; \gamma_{1} \in \mathbb{R}, \gamma_{2} \in \mathbb{R}_{+},\right. \\
& x_{1}^{+} \geq x_{2}^{+} \geq \ldots \geq x_{k}^{+} \geq \ldots \geq 0, x_{1}^{-} \geq x_{2}^{-} \geq \ldots \geq x_{k}^{-} \geq \ldots \geq 0, \\
& \left.\left(x_{1}^{+}\right)^{2}+\left(x_{2}^{+}\right)^{2}+\ldots+\left(x_{k}^{+}\right)^{2}+\ldots+\left(x_{1}^{-}\right)^{2}+\left(x_{2}^{-}\right)^{2}+\ldots+\left(x_{k}^{-}\right)^{2}+\ldots<\infty\right\},
\end{aligned}
$$


such that every central measure $\mu$ can be written as follows:

$$
\mu=\int_{\Delta} \mu^{x} \mathrm{~d} v(x)
$$

where $v$ is a probability measure on $\Delta$. Moreover, for all

$$
x=\left(\gamma_{1}, \gamma_{2}, x_{1}^{+}, x_{2}^{+}, \ldots, x_{k}^{+}, \ldots, x_{1}^{-}, x_{2}^{-}, \ldots, x_{k}^{-}, \ldots\right) \in \Delta
$$

the ergodic measure $\mu^{x}$ can be characterized by its Fourier transform: for all $n \geq 1$ and for any $n \times n$ hermitian matrix $A$,

$$
\int_{\mathcal{H}} \mathrm{e}^{\mathrm{i} \operatorname{Tr}\left[A\left(M_{i j}\right)_{1 \leq i, j \leq n}\right]} \mathrm{d} \mu^{X}(M)=\mathrm{e}^{\mathrm{i} \gamma_{1} \operatorname{Tr}(A)-\gamma_{2} \operatorname{Tr}\left(A^{2}\right) / 2} \operatorname{det}\left[\left(\prod_{k=1}^{\infty} \frac{\mathrm{e}^{-\mathrm{i} x_{k}^{+} A}}{1-\mathrm{i} x_{k}^{+} A}\right)\left(\prod_{k=1}^{\infty} \frac{\mathrm{e}^{\mathrm{i} x_{k}^{-} A}}{1+\mathrm{i} x_{k}^{-} A}\right)\right]
$$

(in this paper, the multiples of identity matrices will sometimes be denoted by complex numbers, and the multiplications by inverse of matrices can be denoted as quotients, when there is no problem of commutativity). Moreover, if $M$ is a random element of $\mathcal{H}$ following the distribution $\mu^{x}$, if $\lambda_{1}^{+}(n) \geq \lambda_{2}^{+}(n) \geq \cdots \geq \lambda_{k}^{+}(n) \geq \cdots \geq 0$ denotes the sequence of positive eigenvalues of the hermitian matrix $\left(M_{i j}\right)_{1 \leq i, j \leq n}$, and if $\lambda_{1}^{-}(n) \leq \lambda_{2}^{-}(n) \leq \cdots \leq$ $\lambda_{k}^{-}(n) \leq \cdots \leq 0$ is the sequence of negative eigenvalues, both sequences being completed by zeros, then the following properties hold almost surely:

(1) For fixed $k \geq 1$ and $n$ going to infinity,

$$
\frac{\lambda_{k}^{+}(n)}{n} \longrightarrow x_{k}^{+} \quad \text { and } \quad \frac{\lambda_{k}^{-}(n)}{n} \longrightarrow-x_{k}^{-}
$$

(2) For $n$ going to infinity,

$$
\frac{1}{n} \sum_{k=1}^{\infty}\left(\lambda_{k}^{+}(n)+\lambda_{k}^{-}(n)\right) \longrightarrow \gamma_{1}
$$

and

$$
\frac{1}{n^{2}} \sum_{k=1}^{\infty}\left(\left(\lambda_{k}^{+}(n)\right)^{2}+\left(\lambda_{k}^{-}(n)\right)^{2}\right) \longrightarrow \gamma_{2}+\sum_{k=1}^{\infty}\left[\left(x_{k}^{+}\right)^{2}+\left(x_{k}^{-}\right)^{2}\right]
$$

Similarly as in the setting of virtual permutations, the first property implies an almost sure convergence for the renormalized extreme eigenvalues of the left-upper blocks of 
any element in $\mathcal{H}$ following a central measure. Borodin and Olshanski [1] construct a remarkable family of central measures, called Hua-Pickrell measures and indexed by a complex parameter $\delta$ whose real part is strictly larger than $-\frac{1}{2}$ (for $\delta \in \mathbb{R}$, see also [4] for such measures on the finite-dimensional unitary group and [11,12] for the Grassmannian case). The Hua-Pickrell measure $m^{(\delta)}$ of parameter $\delta$ is defined as the unique probability measure such that for all $n \geq 1$, the projection $m^{(\delta, n)}$ of $m^{(\delta)}$ on the space of $n \times n$ hermitian matrices satisfies:

$$
m^{(\delta, n)}(d M)=c_{\delta, n} \operatorname{det}\left((1+i M)^{-\delta-n}\right) \operatorname{det}\left((1-i M)^{-\bar{\delta}-n}\right) \prod_{1 \leq j \leq k \leq n} d \mathfrak{R e}\left(M_{j k}\right) \prod_{1 \leq j<k \leq n} d \mathfrak{I m}\left(M_{j k}\right),
$$

where $c_{\delta, n}$ is a normalization constant. As stated above, the measure $m^{(\delta)}$ can be expressed by Equation (1.2). Moreover, Borodin and Olshanski have proved that under $\nu, \gamma_{2}=0$ almost surely, and that the point process $\left\{x_{1}^{+}, x_{2}^{+}, \ldots, x_{k}^{+}, \ldots,-x_{1}^{-},-x_{2}^{-}, \ldots\right.$, $\left.-x_{k}^{-}, \ldots\right\}$ is a determinantal process whose kernel is explicitly expressed in terms of confluent hypergeometric functions.

The similarity between the setting of virtual permutations and the setting of infinite hermitian matrices becomes clearer when one replaces hermitian matrices by unitary matrices, via Cayley transform. More precisely, for all $n \geq 1$, the map

$$
\mathcal{C}_{n}: M \mapsto \frac{M-i}{M+i}
$$

defines a bijection from the set of $n \times n$ hermitian matrices to the set $V(n)$ of $n \times n$ unitary matrices which do not have 1 as an eigenvalue, and the inverse bijection is given by

$$
\mathcal{C}_{n}^{-1}: u \mapsto i \frac{1+u}{1-u} .
$$

For $n \geq m \geq 1$, one defines a natural projection $p_{n, m}$ from the space of $n \times n$ hermitian matrices to the space of $m \times m$ hermitian matrices, simply by taking the left upper block. This projection $p_{n, m}$ induces a map $\tilde{\pi}_{n, m}$ from $V(n)$ to $V(m)$, given by

$$
\tilde{\pi}_{n, m}=\mathcal{C}_{m} \circ p_{n, m} \circ \mathcal{C}_{n}^{-1},
$$

and it is immediate to check that $\tilde{\pi}_{n, p}=\tilde{\pi}_{m, p} \circ \tilde{\pi}_{n, m}$ for $n \geq m \geq p \geq 1$. Moreover, Neretin [9] has computed explicitly the projection $\tilde{\pi}_{n, m}$ : if a matrix

$$
u=\left(\begin{array}{ll}
A & B \\
C & D
\end{array}\right) \in V(n)
$$


is divided into four blocks of sizes $m \times m, m \times(n-m), \quad(n-m) \times m$, and $(n-m) \times(n-m)$, then $1-D$ is invertible and

$$
\tilde{\pi}_{n, m}(u)=A+B(1-D)^{-1} C \in V(m) .
$$

(In fact, the projections defined here are not exactly those which are given in [9]: we have reversed the order of the lines and the columns, and we have changed the matrices to their opposite. These slight changes are made for the coherence of the present paper.) Then, one can define the virtual isometries (or virtual rotations) as the sequences $\left(u_{n}\right)_{n \geq 1}$ of unitary matrices, such that $u_{n} \in V(n)$ for all $n \geq 1$ and $u_{m}=\tilde{\pi}_{n, m}\left(u_{n}\right)$ for all $n \geq m \geq 1$. An equivalent condition is the following: there exists an infinite hermitian matrix $M$ such that for all $n \geq 1$,

$$
u_{n}=\mathcal{C}_{n}\left(\left(M_{i j}\right)_{1 \leq i \leq j \leq n}\right)
$$

If the space of virtual isometries is endowed with the $\sigma$-algebra generated by the coordinates, then the Cayley transform induces a bijection between the probability measures on this space and the probabilities on the space of infinite hermitian matrices, and also a bijection between the central measures: hence, the results of Olshanski and Vershik [10] can be immediately translated into a classification of all the central measures on the space of virtual isometries. One also deduces that under such a central measure, and for all $k \geq 1$, the $k$ th smallest positive and negative eigenangles of $u_{n}$, multiplied by $n$, tend almost surely to a limit when $n$ goes to infinity. The translation of the particular case of Hua-Pickrell measures into the unitary context gives a family $\tilde{\mu}^{(\delta)}$ of central measures on the space of virtual rotations, such that for all $n \geq 1$, its $n$th projection $\tilde{\mu}^{(\delta, n)}$ is given by

$$
\tilde{\mu}^{(\delta, n)}(d u)=c_{\delta, n}^{\prime} \operatorname{det}(1-u)^{\bar{\delta}} \operatorname{det}(1-\bar{u})^{\delta} d u
$$

where $c_{\delta, n}^{\prime}$ is a normalization constant, and $d u$ the Haar measure on $V(n)$, that is the restriction to $V(n)$ of the Haar measure on the unitary group $U(n)$, which is a probability measure since $V(n)$ contains almost every element of $U(n)$. In the case where $\delta=0$, the measure $\tilde{\mu}^{(0)}$ can be called Haar measure on the space of virtual isometries, since for all $n \geq 1$, its $n$th projection is equal to the Haar measure on $V(n)$. Moreover, the corresponding limiting point process of the renormalized scaled eigenangles is a determinantal process with sine kernel: informally, if $x_{1}, \ldots, x_{k} \in \mathbb{R}$, then the probability that there is a point in the neighborhood of $x_{j}$ for all $j \in\{1, \ldots, k\}$ is proportional to $\operatorname{det}\left(K\left(x_{j}, x_{l}\right)_{1 \leq j, l \leq k}\right)$, 
where the kernel $K$ is given by

$$
K(x, y)=\frac{1}{2 \pi} \sin (\pi(x-y)) / \pi(x-y)
$$

Note that the weak version of this result (convergence in law of the point process of the eigenangles toward a sine kernel process) is very classical in random matrix theory. The introduction of the virtual rotations for this problem has the following advantages:

(1) One has a result of almost sure convergence when the dimension goes to infinity, which is quite rare in random matrix theory.

(2) The limit point process is directly associated to a random virtual isometry, with a deterministic map (almost surely well defined): the link between sine kernel process and random matrices is particularly explicit in this setting.

As we have seen in this introduction, there are two kinds of sequences $\left(u_{n}\right)_{n \geq 1}$ of unitary matrices giving a similar behavior for the small eigenangles: the virtual permutations, for which $u_{n}$ is an $n \times n$ permutation matrix (identified with an element of $\mathcal{S}_{n}$ ) for all $n \geq 1$, and the virtual rotations, for which $u_{n} \in V(n)$.

This work is intended as an attempt to understand the links between the virtual permutations and the virtual rotations. Given that the group of permutations of size $n$ can be identified with the corresponding subgroup of permutation matrices of the unitary group, it is natural to expect that virtual permutations can be obtained from the construction of virtual rotations. However, it turns out that one cannot recover the virtual permutations from Neretin's construction because in this construction, for any $n$, 1 cannot be an eigenvalue of $u_{n}$ for any virtual rotation $\left(u_{n}\right)_{n \geq 1}$. We hence propose another construction for virtual isometries, based on complex reflections, which extends both constructions. Our construction provides us with a simple recursive relation between the characteristic polynomials of $u_{n}$ and $u_{n+1}$ for a virtual isometry $\left(u_{n}\right)_{n \geq 1}$. Moreover, it shows us how to generate random virtual isometries. As a consequence, we are able to recover the known result that the smallest normalized eigenangles of a virtual rotation, under the Haar measure, converge almost surely to a point process whose correlation function is given by the sine kernel. Our proof exhibits an interesting interlacement properties for the eigenangles of $u_{n}$ and $u_{n+1}$.

More precisely, in Section 2, we define a projection from $U(n)$ to $U(m)$, which extends both the projection $\pi_{n, m}$ from $\mathcal{S}_{n}$ to $\mathcal{S}_{m}$ and the projection $\tilde{\pi}_{n, m}$ from $V(n)$ to $V(m)$. In Section 3, we deduce in our framework a natural construction of the HuaPickrell measures (and, in particular, the Haar measure) on virtual rotations, in terms of 
products of independent random reflections. As we have seen before, the general results by Borodin and Olshanski [1] and Olshanski and Vershik [10] imply the almost sure convergence of the renormalized eigenangles, for a virtual isometry following Haar measure. In Section 4, we give a direct and purely probabilistic proof of this result, which gives some information on the corresponding rate of convergence.

\section{The Space of Virtual Isometries}

As stated in Section 1, our purpose in this section is to define a strict analog of the virtual permutations, in the context of the unitary group. Our construction is expected to be applicable to other compact groups (like the orthogonal or the symplectic group), however, for the sake of simplicity, we only deal with unitary matrices in this article. The first step of our construction is the following result, which proves that intuitively, it is possible to construct a natural projection from the unitary group of a finite-dimensional Hilbert space $E$ to the unitary group of a subspace $F$ of $E$.

Proposition 2.1. Let $H$ be a complex Hilbert space, $E$ a finite-dimensional subspace of $H$ and $F$ a subspace of $E$. Then, for any unitary operator $u$ on $H$ which fixes each element of $E^{\perp}$, there exists a unique unitary operator $\pi_{E, F}(u)$ on $H$ which satisfies the following two conditions:

(1) $\pi_{E, F}(u)$ fixes each element of $F^{\perp}$;

(2) the image of $H$ by $u-\pi_{E, F}(u)$ is included into the image of $F^{\perp}$ by $u-I d$.

Moreover, if $G$ is a subspace of $F, \pi_{F, G} \circ \pi_{E, F}(u)$ is well defined and is equal to $\pi_{E, G}(u)$.

Proof. Let $x$ be an element of $F \cap(u-\operatorname{Id})\left(F^{\perp}\right)$. There exists $y \in F^{\perp}$ such that $x=u(y)-$ $y$, or equivalently, $u(y)=x+y$. Since $x \in F$ and $y \in F^{\perp}$, one has $\|x\|^{2}+\|y\|^{2}=\|x+y\|^{2}=$ $\|u(y)\|^{2}=\|y\|^{2}$, which implies $x=0$. Now, if $v_{1}$ and $v_{2}$ are two operators which satisfy the conditions defining $\pi_{E, F}(u)$, one has the following:

(1) $v_{1}$ and $v_{2}$ fix globally the space $F$, since they fix the space $F^{\perp}$;

(2) $v_{1}-v_{2}$ vanishes on $F^{\perp}$, since $v_{1}$ and $v_{2}$ fix each point of this space;

(3) the image of $v_{1}-v_{2}$ is included in $(u-\operatorname{Id})\left(F^{\perp}\right)$, since the images of $u-v_{1}$ and $u-v_{2}$ are both included in this space. 
These three properties imply that the image of $v_{1}-v_{2}$ is included in the space $F \cap(u-$ $\operatorname{Id})\left(F^{\perp}\right)=\{0\}$, which proves the uniqueness of $\pi_{E, F}(u)$. Let us now show its existence and the projective property of the map $\pi_{E, F}$.

We can first remark that for $G \subset F \subset E \subset H$, if $\pi_{E, F}$ and $\pi_{F, G}$ are well defined, then $\pi_{E, G}$ is also well defined and is equal to $\pi_{F, G} \circ \pi_{E, F}$. Indeed, for all unitary operators $u$ fixing each element of $E^{\perp}$, the two unitary operators $v:=\pi_{E, F}(u)$ and $w:=\pi_{F, G}(v)$ are well defined and satisfy the following assumptions:

(1) $v$ fixes all the elements of $F^{\perp}$;

(2) $(u-v)(H) \subset(u-\mathrm{Id})\left(F^{\perp}\right)$;

(3) $w$ fixes all the elements of $G^{\perp}$;

(4) $(v-w)(H) \subset(v-\mathrm{Id})\left(G^{\perp}\right)$.

This yields the elementary inclusions

$$
\begin{aligned}
(u-w)(H) & \subset \operatorname{Span}((u-v)(H),(v-w)(H)) \\
& \subset \operatorname{Span}\left((u-\operatorname{Id})\left(F^{\perp}\right),(v-\operatorname{Id})\left(G^{\perp}\right)\right) \\
& \subset \operatorname{Span}\left((u-\operatorname{Id})\left(F^{\perp}\right),(u-\operatorname{Id})\left(G^{\perp}\right),(u-v)\left(G^{\perp}\right)\right) \\
& \subset \operatorname{Span}\left((u-\operatorname{Id})\left(G^{\perp}\right),(u-v)(H)\right) \\
& \subset(u-\operatorname{Id})\left(G^{\perp}\right) .
\end{aligned}
$$

Since $w$ fixes each element of $G^{\perp}, \pi_{E, G}(u)$ is well defined and is equal to $w$. By induction, it is now sufficient to prove the existence of $\pi_{E, F}$ in the particular case where $E=\operatorname{Vect}(F, e)$, where $e$ is a unit vector, orthogonal to $F$. In this case, if $u$ is a unitary operator fixing each element of $E^{\perp}$, then the operator $v:=\pi_{E, F}(u)$ can be constructed explicitly as follows.

(1) If $u(e)=e$, then one takes $v=u$, whch fixes $E^{\perp}$ and $e$, hence, it fixes $F^{\perp}$, and $(u-v)(H)=\{0\}=(u-\operatorname{Id})\left(F^{\perp}\right)$.

(2) If $u(e) \neq e$, then for all $x \in H$, we define

$$
v(x):=u(x)+\frac{\langle e-u(e), u(x)\rangle}{\langle e-u(e), u(e)\rangle}(e-u(e)) .
$$

The denominator in the expression defining $v(x)$ does not vanish and the following properties hold: 
(a) for all $x \in H$,

$$
\begin{aligned}
\|v(x)\|^{2}= & \|u(x)\|^{2}+2 \Re \mathfrak{R e}\left(\frac{|\langle e-u(e), u(x)\rangle|^{2}}{\langle e-u(e), u(e)\rangle}\right) \\
& +\frac{|\langle e-u(e), u(x)\rangle|^{2}}{|\langle e-u(e), u(e)\rangle|^{2}}\|e-u(e)\|^{2} \\
= & \|x\|^{2}+\left(\frac{|\langle e-u(e), u(x)\rangle|^{2}}{|\langle e-u(e), u(e)\rangle|^{2}}\right) \\
& \times\left(2 \mathfrak{R e}(\langle e-u(e), u(e)\rangle)+\|e-u(e)\|^{2}\right) \\
= & \|x\|^{2}+\left(\frac{|\langle e-u(e), u(x)\rangle|^{2}}{|\langle e-u(e), u(e)\rangle|^{2}}\right)\left(\|e\|^{2}-\|u(e)\|^{2}\right) \\
= & \|x\|^{2},
\end{aligned}
$$

which implies that $v$ is a unitary operator;

(b) for $x \in E^{\perp}, u(x)=x$, and $e-u(e) \in E$, since $E$ is globally fixed by $u$. Hence, $\langle e-u(e), u(x)\rangle=0$, and $v(x)=u(x)=x: v$ fixes each element of $E^{\perp}$;

(c) by (2.1), $v$ fixes $e$, and then, it fixes each element of $F^{\perp}$;

(d) Again by (2.1), for all $x \in H, u(x)-v(x)$ is a multiple of $u(e)-e$, and then in the image of $F^{\perp}$ by $u-I d$.

This concludes the proof.

Remark. In the case where $E=\operatorname{Vect}(F, e)$, $e$ being a unit vector, orthogonal to $F$, the formula (2.1) proves that for $e \neq u(e), u=r \pi_{E, F}(u)$, where $r$ is the unique reflection (i.e., $r$ is unitary and $r$ - Id has rank 1) such that $r(e)=u(e)$. Similarly, $u=\pi_{E, F}(u) r^{\prime}$ where $r^{\prime}$ is the unique reflection such that $r^{\prime}\left(u^{-1}(e)\right)=e$.

The following consequence of Proposition 2.1 shows that $\pi_{E, F}$ is also a projection in the sense of the minimization of a distance:

Corollary 2.2. Let $H$ be a complex Hilbert space, and let $U_{0}(H)$ be the space of the unitary operators on $H$ which fix each element of the orthogonal of a finite-dimensional subspace of $H$. Then the map $d$ from $U_{0}(H) \times U_{0}(H)$ to $\mathbb{N}_{0}$, given by $d(u, v):=\operatorname{rank}(u-v)$ defines a finite distance on $U_{0}(H)$. Moreover, if $F \subset E$ are two finite-dimensional subspaces of $H$, and if $u$ is a unitary operator fixing each element of $E^{\perp}$, then $\pi_{E, F}(u)$ is the 
unique unitary operator fixing each element of $F^{\perp}$ and such that $d\left(u, \pi_{E, F}(u)\right)$ is minimal. The image of $H$ by $u-\pi_{E, F}(u)$ is equal to the image of $F^{\perp}$ by $u-I d$, and one has

$$
d\left(u, \pi_{E, F}(u)\right)=\operatorname{dim}(E)-\operatorname{dim}(F)-\operatorname{dim}\left(\left\{x \in E \cap F^{\perp}, u(x)=x\right\}\right),
$$

in particular, if one is not an eigenvalue of the restriction of $u$ to $E \cap F^{\perp}$, then

$$
d\left(u, \pi_{E, F}(u)\right)=\operatorname{dim}(E)-\operatorname{dim}(F) .
$$

Proof. Let $u, v \in U_{0}(H)$. By assumption, the images of $u-\operatorname{Id}$ and $v-\operatorname{Id}$ are finitedimensional, and then $u-v$ has finite rank: $\mathrm{d}(u, v)$ is finite. It is obvious that $d(u, u)=0$ and $d(u, v)=d(v, u)$, and if $d(u, v)=0$, the image of $u-v$ is equal to $\{0\}$, which implies $u=v$. Moreover, if $w \in U_{0}(H)$, then

$$
(u-w)(H) \subset \operatorname{Span}((u-v)(H),(v-w)(H)),
$$

which implies

$$
d(u, w) \leq d(u, v)+d(v, w)
$$

Hence $d$ defines a finite distance. Now, let us suppose that $u$ fixes each element in $E^{\perp}$ and $v$ fixes each element in $F^{\perp}$, for two finite-dimensional spaces $F \subset E$. Then, for $x \in F^{\perp}$, $(u-v)(x)=(u-\operatorname{Id})(x)$, which implies that the image of $u-v$ contains the image of $F^{\perp}$ by $u-I d$. Since by Proposition 2.1, $\left(u-\pi_{E, F}(u)\right)(H) \subset(u-\mathrm{Id})\left(F^{\perp}\right)$ :

(1) $\left(u-\pi_{E, F}(u)\right)(H)=(u-\operatorname{Id})\left(F^{\perp}\right)$ ，

(2) $\pi_{E, F}(u)$ is the unique unitary operator $v$, fixing each element of $F^{\perp}$, and such that the space $(u-v)(H)$, and then the distance $d(u, v)$, is minimal.

Now, since $u$ fixes each element of $E^{\perp},(u-\operatorname{Id})\left(F^{\perp}\right)=(u-\operatorname{Id})\left(E \cap F^{\perp}\right)$, and then

$$
\begin{aligned}
d\left(u, \pi_{E, F}(u)\right) & =\operatorname{dim}\left(E \cap F^{\perp}\right)-\operatorname{dim}\left(\operatorname{Ker}(u-\mathrm{Id}) \cap E \cap F^{\perp}\right) \\
& =\operatorname{dim}(E)-\operatorname{dim}(F)-\operatorname{dim}\left(\left\{x \in E \cap F^{\perp}, u(x)=x\right\}\right),
\end{aligned}
$$

which concludes the proof.

Remark. For other distances $d^{\prime}$ on $U_{0}(H)$, for $F \subset E \subset H, E$ finite-dimensional, and for a unitary operator $u$ fixing all the elements of $E^{\perp}$, it may be possible to define $\pi_{E, F}^{\left(d^{\prime}\right)}(u)$ as the unitary operator $v$ which fixes the elements of $F^{\perp}$ and for which the distance $d^{\prime}(u, v)$ 
is as small as possible. However, for $G \subset E$, one does not have in general $\pi_{E, G}^{\left(d^{\prime}\right)}=\pi_{F, G}^{\left(d^{\prime}\right)} \pi_{E, F}^{\left(d^{\prime}\right)}$. A natural question, not treated here, is the following: what are the distances $d^{\prime}$ for which the projective property $\pi_{E, G}^{\left(d^{\prime}\right)}=\pi_{F, G}^{\left(d^{\prime}\right)} \pi_{E, F}^{\left(d^{\prime}\right)}$ remains true?

The existence of the projective map described above implies the possibility to define the virtual isometries. Indeed, let $H:=\ell^{2}(\mathbb{C})$, and let $\left(e_{n}\right)_{n \geq 1}$ be the canonical Hilbert basis of $H$. For all $n \geq 1$, the space of unitary operators fixing each element of the orthogonal of $\operatorname{Span}\left(e_{1}, \ldots, e_{n}\right)$ can be canonically identified with the unitary group $U(n)$. By this identification, for $n \geq m \geq 1$, the projection $\pi_{\operatorname{Span}\left(e_{1}, \ldots, e_{n}\right), \operatorname{Span}\left(e_{1}, \ldots, e_{m}\right)}$ defines a map $\pi_{n, m}$ from $U(n)$ to $U(m)$, and for $n \geq m \geq p \geq 1$, one has $\pi_{n, p}=\pi_{m, p} \circ \pi_{n, m}$.

Definition 2.3. A virtual isometry is a sequence $\left(u_{n}\right)_{n \geq 1}$ of unitary matrices, such that for all $n \geq 1, u_{n} \in U(n)$ and $\pi_{n+1, n}\left(u_{n+1}\right)=u_{n}$. In this case, for all $n \geq m \geq 1, \pi_{n, m}\left(u_{n}\right)=u_{m}$. The space of virtual isometries will be denoted $U^{\infty}$.

Remark. Given two virtual isometries $\left(u_{n}\right)_{n \geq 1}$ and $\left(v_{n}\right)_{n \geq 1}$, the sequence $\left(w_{n}\right)_{n \geq 1}$ obtained from pointwise multiplication $w_{n}=u_{n} v_{n}$ is not a virtual isometry in general: the coherence property does not hold for $\left(w_{n}\right)_{n \geq 1}$. Hence $U^{\infty}$ has no group structure.

It is now possible to check that the virtual isometries defined in the present paper are both a generalization of the virtual permutations, and an extension of the virtual isometries in the sense of Neretin.

Proposition 2.4. Let $\left(\sigma_{n}\right)_{n \geq 1}$ be a sequence of permutations such that $\sigma_{n} \in \mathcal{S}_{n}$ for all $n \geq 1$, and let $\left(\Sigma_{n}\right)_{n \geq 1}$ be the corresponding sequence of permutation matrices. Then $\left(\Sigma_{n}\right)_{n \geq 1}$ is a virtual isometry if and only if $\left(\sigma_{n}\right)_{n \geq 1}$ is a virtual permutation.

Proof. In this proof, and in all the sequel of the article, we identify $\mathbb{C}^{n}$ with the set of the sequences $\left(x_{k}\right)_{k \geq 1}$ such that $x_{k}=0$ for all $k>n$, and we define $\left(e_{k}\right)_{k \geq 1}$ as the canonical basis of $\mathbb{C}^{\mathbb{N}}$ : in this way, $\left(e_{k}\right)_{1 \leq k \leq n}$ is identified with the canonical basis of $\mathbb{C}^{n}$ for all $n \geq 1$. With this convention, the sequence $\left(\Sigma_{n}\right)_{n \geq 1}$ is a virtual isometry if and only if for all $n \geq 1$, the image of $\Sigma_{n+1}-\Sigma_{n}$ is in the vector space generated by $e_{n+1}-\Sigma_{n+1}\left(e_{n+1}\right)=e_{n+1}-e_{\sigma_{n+1}(n+1)}$. Since $\Sigma_{n+1}\left(e_{j}\right)-\Sigma_{n}\left(e_{j}\right)=e_{\sigma_{n+1}(j)}-e_{\sigma_{n}(j)}$, the condition above is satisfied if and only if for all $n \geq 1, j \in\{1, \ldots, n\}$, one of the two following situations arises:
(1) $\sigma_{n+1}(j)=\sigma_{n}(j)$;
(2) $\sigma_{n+1}(j)=n+1$ and $\sigma_{n}(j)=\sigma_{n+1}(n+1)$. 
In other words, $\left(\Sigma_{n}\right)_{n \geq 1}$ is a virtual isometry if and only if for all $n \geq 1$, one of the two following cases holds:

(1) the restriction of $\sigma_{n+1}$ to $\{1, \ldots, n\}$ is equal to $\sigma_{n}$, and $\sigma_{n+1}(n+1)=n+1$;

(2) $\sigma_{n+1}(n+1) \neq n+1, \sigma_{n}(j)=\sigma_{n+1}(j)$ for $j \in\{1, \ldots, n\} \backslash\left\{\sigma_{n+1}^{-1}(n+1)\right\}$, and $\sigma_{n}(j)=$ $\sigma_{n+1}(n+1)$ for $j=\sigma_{n+1}^{-1}(n+1)$.

This is equivalent to the fact that $\left(\sigma_{n}\right)_{n \geq 1}$ is a virtual permutation.

Proposition 2.5. Let $\left(u_{n}\right)_{n \geq 1}$ be a sequence of unitary matrices such that $u_{n} \in V(n)$ for all $n \geq 1$ (recall that $V(n)$ is the set of $n \times n$ unitary matrices which do not have one as an eigenvalue). Then, $\left(u_{n}\right)_{n \geq 1}$ is a virtual rotation in the sense of Neretin if and only if it is a virtual isometry in the sense of Definition 2.3.

Proof. It is sufficient to check that for $n \geq m \geq 1$, and $u_{n} \in V(n), \pi_{n, m}\left(u_{n}\right)=\tilde{\pi}_{n, m}\left(u_{n}\right)$, where $\tilde{\pi}_{n, m}$ is defined as in Section 1. By Corollary 2.2, one deduces that it is sufficient to bound the rank of

$$
R:=u_{n}-\left(\begin{array}{cc}
\tilde{\pi}_{n, m}\left(u_{n}\right) & 0 \\
0 & \operatorname{Id}_{n-m}
\end{array}\right)
$$

by $n-m$, since one is not an eigenvalue of $u_{n}$. Now, if $u_{n}$ is divided into blocks of size $m \times m, m \times(n-m),(n-m) \times m,(n-m) \times(n-m):$

$$
u_{n}=\left(\begin{array}{ll}
A & B \\
C & D
\end{array}\right)
$$

then

$$
\begin{aligned}
R & =\left(\begin{array}{ll}
A & B \\
C & D
\end{array}\right)-\left(\begin{array}{cc}
A+B(1-D)^{-1} C & 0 \\
0 & 1
\end{array}\right) \\
& =\left(\begin{array}{cc}
B(D-1)^{-1} C & B \\
C & D-1
\end{array}\right)=\left(\begin{array}{c}
B(D-1)^{-1} \\
1
\end{array}\right)\left(\begin{array}{ll}
C & D-1
\end{array}\right) .
\end{aligned}
$$

In other words, $R$ is the product of a $n \times(n-m)$ matrix by a $(n-m) \times n$ matrix: its rank cannot be strictly larger than $n-m$. 
Now, since the virtual isometries are the natural generalizations of the virtual permutations, it is natural to ask if there is an analog of the Chinese restaurant process. The answer is positive:

Proposition 2.6. Let $\left(x_{n}\right)_{n \geq 1}$ be a sequence of vectors, $x_{n}$ lying on the complex unit sphere of $\mathbb{C}^{n}$ for all $n \geq 1$. Then, there exists a unique virtual isometry $\left(u_{n}\right)_{n \geq 1}$ such that $u_{n}\left(e_{n}\right)=x_{n}$ for all $n \geq 1$, and $u_{n}$ is given by

$$
u_{n}=r_{n} r_{n-1} \cdots r_{1},
$$

where for $j \in\{1, \ldots, n\}, r_{j}=$ Id if $x_{j}=e_{j}$, and otherwise, $r_{j}$ is the unique reflection such that $r_{j}\left(e_{n}\right)=x_{n}$. Moreover, in the particular case where for all $n \geq 1, x_{n}=e_{i_{n}}$ for $i_{n} \in\{1, \ldots, n\}$, then $\left(u_{n}\right)_{n \geq 1}$ is the sequence of matrices associated to a virtual permutation $\left(\sigma_{n}\right)_{n \geq 1}$ constructed by the Chinese restaurant process: for all $n \geq 1$,

$$
\sigma_{n}=\tau_{n, i_{n}} \tau_{n-1, i_{n-1}} \cdots \tau_{1, i_{1}}
$$

where, for $j, k \in\{1, \ldots, n\}, \tau_{j, k}=\operatorname{Id}$ if $j=k$ and $\tau_{j, k}$ is the transposition $(j, k)$ if $j \neq k$.

Proof. One has $u_{1}\left(e_{1}\right)=x_{1}$ if and only if $u_{1}=x_{1}$, which is equal to $r_{1}$. For all $n \geq 1$, two cases are possible:

(1) if $x_{n+1}=e_{n+1}$, then $\pi_{n+1, n}\left(u_{n+1}\right)=u_{n}$ and $u_{n+1}\left(e_{n+1}\right)=e_{n+1}$ if and only if $u_{n+1}=$ $\left(u_{n}\right) \oplus 1$, where the symbol $\oplus$ denotes diagonal blocks of matrices;

(2) if $x_{n+1} \neq e_{n+1}$, Equation (2.1) and the remark after the proof of Proposition 2.1 imply that $\pi_{n+1, n}\left(u_{n+1}\right)=u_{n}$ and $u_{n+1}\left(e_{n+1}\right)=x_{n+1}$ if and only if $u_{n+1}=r_{n+1}$ $\left(u_{n} \oplus 1\right)$.

By induction, the uniqueness and the general form of $u_{n}$ is proved. If $x_{n}=e_{i_{n}}$ for all $n \geq 1, r_{n}$ is the matrix of the permutation $\tau_{n, i_{n}}$, which easily implies the second part of Proposition 2.6.

The construction given in Proposition 2.6 implies, in particular, that the space $U^{\infty}$ is not empty. Moreover, it is possible to use it to define probability measures on this space. 


\section{Some Remarkable Measures on $U^{\infty}$}

Once the space $U^{\infty}$ is constructed, it is natural to ask if there exists an analog of the Haar measure on this space. As seen in Section 1, the positive answer can be deduced from the results given in $[1,9,10]$, and of the fact that under Haar measure on $U(n)$, almost every matrix is in $V(n)$. A more direct proof can be easily deduced from the results given by Bourgade et al. [2]:

Proposition 3.1. Let $\left(x_{n}\right)_{n \geq 1}$ be a random sequence of vectors, $x_{n}$ lying on the complex unit sphere of $\mathbb{C}^{n}$ for all $n \geq 1$, and let $\left(u_{n}\right)_{n \geq 1}$ be the unique virtual isometry such that $u_{n}\left(e_{n}\right)=x_{n}$ for all $n \geq 1$. Then, for each $n$, the matrix $u_{n}$ follows the Haar measure on $U(n)$ if and only if $x_{1}, \ldots, x_{n}$ are independent and for all $j \in\{1,2, \ldots, n\}, x_{j}$ follows uniform measure on the complex unit sphere of $\mathbb{C}^{n}$.

This result has the following consequence, showing the compatibility between the Haar measure on $U(n), n \geq 1$ and the projections $\pi_{n, m}, n \geq m \geq 1$.

Corollary 3.2. For all $n \geq m \geq 1$, the image of the Haar measure on $U(n)$ by the projection $\pi_{n, m}$ is equal to the Haar measure on $U(m)$.

Remark. The statement given in Corollary 3.2 is meaningful only if the application $\pi_{n, m}$ is measurable with respect to the Borel $\sigma$-algebras of $U(n)$ and $U(m)$. This fact can be easily checked by using the formula (2.1). Moreover, Corollary 3.2 can be easily proved directly. Indeed, let $n \geq 1$, let $u$ be a matrix on $U(n+1)$ following the Haar measure, and let $a$ be a deterministic matrix on $U(n)$. The invariance of the Haar measure implies that $u(a \oplus 1)$ follows the Haar measure on $U(n+1)$, and then has the same law as $u$. Now, it is easy to check that $\pi_{n+1, n}(u(a \oplus 1))=\pi_{n+1, n}(u) a$, hence, $\pi_{n+1, n}(u) a$ has the same law as $\pi_{n+1, n}(u)$ for all $a \in U(n)$. One deduces that $\pi_{n+1, n}(u)$ follows the Haar measure on $U(n)$.

The property of compatibility given in Corollary 3.2 implies the possibility to define the Haar measure on $U^{\infty}$. In order to do this properly, let us prove the following result, about the extension of measures:

Lemma 3.3. Let $\mathcal{U}$ be the $\sigma$-algebra on $U^{\infty}$, generated by the sets:

$$
\left\{\left(u_{n}\right)_{n \geq 1}, u_{k} \in B_{k}\right\},
$$


for all $k \geq 1$ and for all Borel sets $B_{k}$ in $U(k)$. Let $\left(\mu_{n}\right)_{n \geq 1}$ be a family of probability measures, $\mu_{n}$ defined on the space $U(n)$ (endowed with its Borel $\sigma$-algebra), and such that the image of $\mu_{n+1}$ by $\pi_{n+1, n}$ is equal to $\mu_{n}$ for all $n \geq 1$. Then, there exists a unique probability measure on $\left(U^{\infty}, \mathcal{U}\right)$ such that its image by the $n$th coordinate is equal to $\mu_{n}$ for all $n \geq 1$.

Proof. For any element $\left(u_{n}\right)_{n \geq 1}$ in $U^{\infty}, u_{p}$ can be expressed as a Borel function of $u_{m}$ for all $m \geq p \geq 1$. One deduces that the family of sets of the form

$$
\left\{\left(u_{n}\right)_{n \geq 1}, u_{k} \in B_{k}\right\}
$$

is stable by finite intersection. This implies the uniqueness part of Lemma 3.3, by the monotone class theorem. In order to prove the existence, let us consider the product $V$ of all the unitary groups $U(n), n \geq 1$, endowed with the product $\mathcal{V}$ of their Borel $\sigma$-algebras. For all $n \geq 1$, let us define the measure $\tilde{\mu}_{n}$ on the space $U(1) \times \cdots \times U(n)$, endowed with the corresponding product of Borel $\sigma$-algebras, as the image of $\mu_{n}$ by the map:

$$
u_{n} \mapsto\left(\pi_{n, 1}\left(u_{n}\right), \ldots, \pi_{n, n-1}\left(u_{n}\right), \pi_{n, n}\left(u_{n}\right)\right)
$$

from $U(n)$ to $U(1) \times \cdots \times U(n)$. The projective property of $\pi_{m, p}, m \geq p \geq 1$ and the fact that $\mu_{n}$ is the image of $\mu_{n+1}$ by $\pi_{n+1, n}$ implies that for all $n \geq 1$, the restriction of $\tilde{\mu}_{n+1}$ to the $n$ first coordinates is equal to $\tilde{\mu}_{n}$. The classical theorem of extension of probability measures implies that there exists a measure $\tilde{\mu}$ on $(V, \mathcal{V})$ such that its restriction to the $n$ first coordinates is equal to $\tilde{\mu}_{n}$, for all $n \geq 1$. By construction, $\tilde{\mu}$ is carried by the set $U^{\infty}$, which is in $\mathcal{V}$, hence, it induces a measure on $U^{\infty}$, endowed with the intersection of $\mathcal{V}$ and $\mathcal{P}\left(U^{\infty}\right)$, which is equal to $\mathcal{U}$.

By combining Proposition 3.1 and Lemma 3.3, one deduces the existence of the Haar measure on virtual isometries:

Proposition 3.4. There exists a unique probability measure $\mu^{(0)}$ on the space $\left(U^{\infty}, \mathcal{U}\right)$ such that its image by all the coordinate maps are equal to the Haar measure on the corresponding unitary group. This measure can be described as follows. Let $\left(x_{n}\right)_{n \geq 1}$ be a random sequence of vectors, $x_{n}$ lying on the complex unit sphere of $\mathbb{C}^{n}$ for all $n \geq 1$, and let $\left(u_{n}\right)_{n \geq 1}$ be the unique virtual isometry such that $u_{n}\left(e_{n}\right)=x_{n}$ for all $n \geq 1$. Then, the 
distribution of $\left(u_{n}\right)_{n \geq 1}$ is equal to $\mu^{(0)}$ if and only if $\left(x_{n}\right)_{n \geq 1}$ are independent, and for all $n \geq 1, x_{n}$ follows the uniform measure on the complex unit sphere of $\mathbb{C}^{n}$.

The Haar measure $\mu^{(0)}$ is the analog of the uniform measure on virtual permutations, which can be obtained in the setting of Proposition 3.4, by taking $\left(x_{n}\right)_{n \geq 1}$ independent, $x_{n}$ uniform on the finite set $\left\{e_{1}, \ldots, e_{n}\right\}$. Moreover, it is possible to generalize the Haar measure on virtual isometries, in the same way as uniform measure on virtual permutations can be generalized by considering the Ewens measures. Enouncing this generalization requires the so-called $h$-sampling (or $h$-transform), which can be described as follows. Let $(X, \mathcal{F}, \mu)$ be a probability space. For a given measurable function $h: X \mapsto \mathbb{R}^{+}$ such that $0<\mathbb{E}_{\mu}(h)<\infty$, a probability measure $\mu^{\prime}$ is said to be the $h$-sampling of $\mu$ if and only if for all bounded measurable functions $f$,

$$
\mathbb{E}_{\mu^{\prime}}(f)=\frac{\mathbb{E}_{\mu}(f h)}{\mathbb{E}_{\mu}(h)}
$$

Here, for all $n \geq 1$, and for $\delta \in \mathbb{C}$ such that $\mathfrak{R e}(\delta)>-\frac{1}{2}$, it is possible to define a probability measure $v_{\delta}^{(n)}$ as the $h$-sampling of the uniform measure on the complex unit sphere, for

$$
h(x)=\left(1-\left\langle e_{n}, x\right\rangle\right)^{\bar{\delta}}\left(1-\overline{\left\langle e_{n}, x\right\rangle}\right)^{\delta},
$$

where the imaginary part of the logarithm of $1-\left\langle e_{n}, x\right\rangle$ is taken in the interval $(-\pi / 2, \pi / 2)$. Then, Bourgade et al. [2] have essentially proved the following result:

Proposition 3.5. Let $\left(x_{n}\right)_{n \geq 1}$ be a random sequence of independent vectors such that for all $n \geq 1, x_{n}$ follows the distribution $v_{\delta}^{(n)}$ on the complex unit sphere of $\mathbb{C}^{n}$ for all $n \geq 1$, and let $\left(u_{n}\right)_{n \geq 1}$ be the unique virtual isometry such that $u_{n}\left(e_{n}\right)=x_{n}$ for all $n \geq 1$. Then, for all $n \geq 1$, the distribution of $u_{n}$ can be described as the $h$-sampling of the Haar measure on $U(n)$, where the function $h$ is given by

$$
h(u)=\operatorname{det}(\operatorname{Id}-u)^{\bar{\delta}} \operatorname{det}(\operatorname{Id}-\bar{u})^{\delta},
$$

where the logarithm of $\operatorname{det}(\operatorname{Id}-u)$ is taken in the unique way such that it is continuous on the connected set $\{u \in U(n), \operatorname{det}(\operatorname{Id}-u) \neq 0\}$, and real (equal to $n \log (2))$ for $u=-\operatorname{Id}$. This construction determines a measure $\mu^{(\delta)}$ on the space $\left(U^{\infty}, \mathcal{U}\right)$, which can be identified with the Hua-Pickrell measure $\tilde{\mu}^{(\delta)}$ given in Section 1. 
The Hua-Pickrell measures are also analogs of Ewens measures on the space of virtual permutations. Indeed, the Ewens measure of parameter $\theta>0$, on the space of virtual permutations, can be constructed in our framework by taking $\left(x_{n}\right)_{n \geq 1}$ independent and for all $n \geq 1$ :

(1) $x_{n} \in\left\{e_{1}, \ldots, e_{n}\right\}$ almost surely;

(2) $\mathbb{P}\left[x_{n}=e_{n}\right]=\theta /(\theta+n-1)$;

(3) For all $j \in\{1, \ldots, n-1\}, \mathbb{P}\left[x_{n}=e_{j}\right]=1 /(\theta+n-1)$.

The law of $x_{n}$ can be viewed as an $h$-sampling of the uniform measure on the space $\left\{e_{1}, \ldots, e_{n}\right\}$, where the function $h$ can be written

$$
h(x)=\left(1+\left\langle e_{n}, x\right\rangle\right)^{2 \delta},
$$

for $\delta=\log \theta / \log 4$, the only difference between Equations (3.1) and (3.2) is the sign change. Now, if $n \geq 1$ and if $v$ is a probability measure on $U(n)$ such that $u \mapsto \mid \log \operatorname{det}(\operatorname{Id}-$ $u) \mid$ is integrable with respect to $v$ (with the same convention for the logarithm as in Proposition 3.5), let us define the capacity (By analogy with the multiple input multiple output (MIMO) systems, where the capacity is $\operatorname{det}\left(\operatorname{Id}+H\left({ }^{\mathrm{t}} H\right)\right)$, where $H$ is the rectangular transmission matrix.) of $v$ as the expectation of $\log \operatorname{det}(\operatorname{Id}-u), u \in U(n)$ following the distribution $v$. A striking fact about the finite-dimensional projections of the Hua-Pickrell distributions is that they maximize the entropy among all the probabilities which have the same capacity. A similar result has already been proved in [15] in the context of permutation groups: if the order of the group and the average number of cycles are fixed, then there exists a unique measure which has the largest entropy, and this measure is the Ewens measure with a suitable parameter. The equivalent result for unitary matrices is the following:

Proposition 3.6. Let $n \geq 1$, and let $\delta \in \mathbb{C}$ be such that $\mathfrak{R e}(\delta)>-\frac{1}{2}$. Then the capacity $C_{n}(\delta)$ of $\mu^{(\delta, n)}$, the projection on $U(n)$ of the Hua-Pickrell measure of parameter $\delta$, is well defined. Moreover, if $f$ denotes the density of $\mu^{(\delta, n)}$ with respect to the Haar measure, if $v$ is a measure on $U(n)$ which is absolutely continuous with respect to the Haar measure, with density $g$, and if the capacity of $v$ is well defined and equal to $C_{n}(\delta)$, then the entropy of $g$ is smaller than or equal to the entropy of $f$, that is, the integral of $-g \log g$ with respect to the Haar measure (which is well defined in $\mathbb{R} \cup\{-\infty\}$ ) is smaller than or equal to the integral of $-f \log f$, which is finite. The equality holds only if $f=g$ almost everywhere, that is, if $v$ is equal to $\mu^{(\delta, n)}$. 
Proof. The integrability condition is equivalent to the fact that, for $u \in U(n)$ following the Haar measure:

$$
\mathbb{E}\left[|\log \operatorname{det}(\operatorname{Id}-u)| \operatorname{det}(\operatorname{Id}-u)^{\bar{\delta}} \operatorname{det}(\operatorname{Id}-\bar{u})^{\delta}\right]<\infty .
$$

By the results of Bourgade et al. [2], it is equivalent to prove that:

$$
\left.\mathbb{E}\left[\mid \sum_{k=1}^{n} \log \left(1-\left\langle e_{k}, x_{k}\right\rangle\right) \prod_{k=1}^{n}\left(1-\left\langle e_{k}, x_{k}\right\rangle\right)^{\bar{\delta}}\left(1-\overline{\left\langle e_{k}, x_{k}\right.}\right\rangle\right)^{\delta} \mid\right]<\infty,
$$

where the $\left(x_{k}\right)_{1 \leq k \leq n}$ are independent, $x_{k}$ uniform on the complex unit sphere of $\mathbb{C}^{k}$. Then, it is sufficient to have, for all $k \in\{1, \ldots, n\}$ :

$$
\mathbb{E}\left[\left|\left(1-\left\langle e_{k}, x_{k}\right\rangle\right)^{\bar{\delta}}\left(1-\overline{\left\langle e_{k}, x_{k}\right\rangle}\right)^{\delta}\right|\right]<\infty, \mathbb{E}\left[\left|\log \left(1-\left\langle e_{k}, x_{k}\right\rangle\right)\left(1-\left\langle e_{k}, x_{k}\right\rangle\right)^{\bar{\delta}}\left(1-\overline{\left\langle e_{k}, x_{k}\right\rangle}\right)^{\delta}\right|\right]<\infty .
$$

These integrability conditions are implied by

$$
\mathbb{E}\left[\left|1-\left\langle e_{k}, x_{k}\right\rangle\right|^{2 \mathfrak{R e}(\delta)}\right]<\infty, \mathbb{E}\left[|\log | 1-\left\langle e_{k}, x_{k}\right\rangle||\left|1-\left\langle e_{k}, x_{k}\right\rangle\right|^{2 \Re \mathfrak{R}(\delta)}\right]<\infty,
$$

and one checks that these conditions are satisfied for all $\delta \in \mathbb{C}^{*}$ such that $\mathfrak{R e}(\delta)>-\frac{1}{2}$. If $f$ and $g$ are defined as in Proposition 3.6, the integrability of $f \log f$ under the Haar measure, which implies the finiteness of the entropy of $\mu_{\delta}^{(n)}$, can be proved in a similar way.

Concerning the optimality to be proved, it results from the elementary inequality

$$
g \log g>f \log f+(g-f)(1+\log f),
$$

for all positive $f \neq g$, as shown by a direct study of the function $f \mapsto f \log f+(g-f)(1+$ $\log f$ ). One deduces that under the Haar measure, and for $f$ not almost everywhere equal to $g$,

$$
\mathbb{E}[-g \log g]<\mathbb{E}[-f \log f]+\mathbb{E}[(g-f)(1+\log f)]
$$

Moreover, as $v$ and $\mu^{(\delta, n)}$ have the same capacity, the expectation $\mathbb{E}[(g-f)(1+\log f)]$ is well defined and equal to zero: it is also $\mathbb{E}[(g-f) \log f]$ (because $\mathbb{E}(f)=\mathbb{E}(g)=1$ for probability densities), which is exactly a multiple of the difference of the capacities, thanks to the particular form of $\log f(u)=(\delta+\bar{\delta}) \operatorname{det}(\operatorname{Id}-u)$. 
Now, in the next section, we go back to virtual isometries and we prove a strong convergence result for their eigenangles.

\section{Strong Convergence of the Eigenangles}

As it was seen above, virtual isometries provide us with the possibility to define on the same probability space random matrix models for all the finite dimensions. It is then possible to prove strong results, that is, properties of almost sure convergence when the dimension goes to infinity. In the particular case of virtual permutations, Tsilevich [15] proved the following result:

Theorem 4.1. Let $\sigma=\left(\sigma_{n}\right)_{n \geq 1}$ be a virtual permutation following the Ewens measure of parameter $\theta>0$, and for $n \geq 1, p \geq 1$, let $\ell_{p}\left(\sigma_{n}\right)$ be the length of the $p$ th longest cycle of the permutation $\sigma_{n} \in \mathcal{S}_{n}$ (for $p$ larger than the number of cycles of $\sigma_{n}$, one defines $\ell_{p}\left(\sigma_{n}\right):=0$ ). Then, almost surely, for all $p \geq 1$, the limit:

$$
Y_{p}(\sigma):=\lim _{n \rightarrow \infty} \frac{\ell_{p}\left(\sigma_{n}\right)}{n}
$$

exists, and $\left(y_{p}(\sigma)\right)_{p \geq 1}$ follows a Poisson-Dirichlet distribution of parameter $\theta$. (For explicit formulas and characterizations of Poisson-Dirichlet distributions, see [13].)

From Theorem 4.1, it is not difficult to deduce the following result, giving an almost sure convergence for the eigenangles of the sequence of permutations matrices associated to a virtual permutation.

Corollary 4.2. Let $\left(u_{n}\right)_{n \geq 1}$ be a random virtual isometry consisting of the sequence of permutation matrices associated to a virtual permutation $\sigma$ which follows the Ewens measure of parameter $\theta>0$. Then, for all $n \geq 1$, zero is an eigenangle of $u_{n}$, and its multiplicity increases almost surely to infinity when $n$ goes to infinity. Moreover, for $n \geq 1$, $k \geq 1$, let $\theta_{k}^{(n)}$ be the $k$ th smallest strictly positive eigenangle of $u_{n}$, and $\theta_{-k}^{(n)}$ the $k$ th largest strictly negative eigenangle of $u_{n}$. Then, almost surely, for all $n \geq 1, k \geq 1, \theta_{-k}^{(n)}=-\theta_{k}^{(n)}$, and for $n$ going to infinity, $n \theta_{k}^{(n)} / 2 \pi$ converges to the $k$ th smallest element of the set which contains exactly all the strictly positive multiples of $1 / y_{p}(\sigma)$ for all $p \geq 1$, where $y_{p}(\sigma)$ is defined in Theorem 4.1. 
In this section, we give a direct and purely probabilistic proof of an analog of Corollary 4.2 for random virtual isometries which follow the Haar measure.

Theorem 4.3. Let $\left(u_{n}\right)_{n \geq 1}$ be a random virtual isometry, following the Haar measure. For $n \geq 1, k \geq 1$, let $\theta_{k}^{(n)}$ be the $k$ th smallest strictly positive eigenangle of $u_{n}$, and let $\theta_{1-k}^{(n)}$ be the $k$ th largest nonnegative eigenangle of $u_{n}$. Then almost surely, for all $k \in \mathbb{Z}, n \theta_{k}^{(n)} / 2 \pi$ converges to a limit $x_{k}$ when $n$ goes to infinity, with the following rate:

$$
n \theta_{k}^{(n)} / 2 \pi=x_{k}+O\left(n^{-\varepsilon}\right)
$$

for some universal constant $\varepsilon>0$. Moreover, the point process $\left(x_{k}\right)_{k \in \mathbb{Z}}$ is a determinantal process and its kernel $K$ is the sine kernel, that is, it is given by

$$
K(x, y)=\frac{\sin (\pi(x-y))}{\pi(x-y)} .
$$

In the proof of Theorem 4.3, the first step is to find an explicit relation between the characteristic polynomials of $u_{n}$ and $u_{n+1}$, when $\left(u_{n}\right)_{n \geq 1}$ is a virtual isometry.

Lemma 4.4. Let $\left(u_{n}\right)_{n \geq 1}$ be a virtual isometry, and for $n \geq 1$, let $x_{n}:=u_{n}\left(e_{n}\right), v_{n}:=x_{n}-e_{n}$, let $\left(f_{k}^{(n)}\right)_{1 \leq k \leq n}$ be an orthonormal basis of $\mathbb{C}^{n}$, consisting of eigenvectors of $u_{n}$, let $\left(\lambda_{k}^{(n)}\right)_{1 \leq k \leq n}$ be the corresponding sequence of eigenvalues, let $P_{n}$ be the characteristic polynomial of $u_{n}$, given by

$$
P_{n}(z):=\operatorname{det}\left(z \operatorname{Id}_{n}-u_{n}\right),
$$

and let us decompose the vector $x_{n+1} \in \mathbb{C}^{n+1}$ as follows:

$$
x_{n+1}=\sum_{k=1}^{n} \mu_{k}^{(n)} f_{k}^{(n)}+v_{n} e_{n+1} .
$$

Then for all $n \geq 1$ such that $x_{n+1} \neq e_{n+1}$, one has $v_{n} \neq 1$, and the polynomials $P_{n}$ and $P_{n+1}$ satisfy the relation:

$$
P_{n+1}(z)=\frac{P_{n}(z)}{\bar{v}_{n}-1}\left[\left(z-v_{n}\right)\left(\bar{v}_{n}-1\right)-(z-1) \sum_{k=1}^{n}\left|\mu_{k}^{(n)}\right|^{2} \frac{\lambda_{k}^{(n)}}{z-\lambda_{k}^{(n)}}\right],
$$

for all $z \notin\left\{\lambda_{1}^{(n)}, \ldots, \lambda_{n}^{(n)}\right\}$. 
Proof. Since $\left(u_{n}\right)_{n \geq 1}$ is a virtual isometry and $x_{n+1} \neq e_{n+1}$, one has $u_{n+1}=r_{n+1}\left(u_{n} \oplus 1\right)$, where $r_{n+1}$ is the unique reflection such that $r_{n+1}\left(e_{n+1}\right)=x_{n+1}$. One can check that the matrix $r_{n+1}$ is given by:

$$
r_{n+1}=\operatorname{Id}_{n+1}+\frac{1}{\bar{v}_{n}-1} v_{n+1} \bar{v}_{n+1}^{t}
$$

which implies, for $z \notin\left\{\lambda_{1}^{(n)}, \ldots \lambda_{n}^{(n)}, 1\right\}$,

$$
\begin{aligned}
P_{n+1}(z) & =\operatorname{det}\left(z \operatorname{Id}_{n+1}-u_{n} \oplus 1\right) \operatorname{det}\left[\operatorname{Id}_{n+1}-\left(\frac{1}{\bar{v}_{n}-1}\left(z \operatorname{Id}_{n+1}-u_{n} \oplus 1\right)^{-1} v_{n+1} \bar{v}_{n+1}^{t}\left(u_{n} \oplus 1\right)\right)\right] \\
& =(z-1) P_{n}(z)\left[1-\frac{1}{\bar{v}_{n}-1} \operatorname{Tr}\left(\left(z \operatorname{Id}_{n+1}-u_{n} \oplus 1\right)^{-1} v_{n+1} \bar{v}_{n+1}^{t}\left(u_{n} \oplus 1\right)\right)\right],
\end{aligned}
$$

since $\operatorname{det}(\operatorname{Id}+A)=1+\operatorname{Tr}(A)$ for any matrix $A$ with rank 1 . One deduces, by writing the matrices in the basis $\left(e_{n+1}, f_{1}^{(n)}, \ldots, f_{n}^{(n)}\right)$ :

$$
P_{n+1}(z)=(z-1) P_{n}(z)\left[1-\frac{1}{\bar{v}_{n}-1}\left(\frac{\left|v_{n}-1\right|^{2}}{z-1}+\sum_{k=1}^{n}\left|\mu_{k}^{(n)}\right|^{2} \frac{\lambda_{k}^{(n)}}{z-\lambda_{k}^{(n)}}\right)\right],
$$

which implies Lemma 4.4 for $z \neq 1$. The case $z=1$ can then be deduced from the fact that $P_{n}$ and $P_{n+1}$ are polynomial functions.

From Lemma 4.4, it is possible to deduce some information on the behavior of the eigenangles corresponding to a virtual isometry:

Lemma 4.5. Let $\left(u_{n}\right)_{n \geq 1}$ be a virtual isometry, such that, with the notation of Theorem 4.3 and Lemma 4.4, the event $E_{0}:=\left\{\theta_{0}^{(1)} \neq 0\right\} \cap\left\{\forall n \geq 1, v_{n} \neq 0\right\} \cap\{\forall n \geq 1, k \in$ $\left.\{1,2, \ldots, n\}, \mu_{k}^{(n)} \neq 0\right\}$ holds. Then, for all $n \geq 1, k \in\{1, \ldots, n\}$,

$$
\begin{aligned}
\rho_{n} & :=\left|v_{n}\right| \in(0,1), \\
\psi_{n} & :=\operatorname{Arg}\left(v_{n}\right) \in(-\pi, \pi], \\
\gamma_{k}^{(n)} & :=\frac{\left|\mu_{k}^{(n)}\right|^{2}}{1-\left|v_{n}\right|^{2}}
\end{aligned}
$$


are well defined, and $\sum_{k=1}^{n} \gamma_{k}^{(n)}=1$. Moreover, for all $n \geq 1$, the expression

$$
\Phi(\eta):=\left(1+\rho_{n}^{2}\right) \cos (\eta / 2)-2 \rho_{n} \cos \left(\eta / 2-\psi_{n}\right)+\left(1-\rho_{n}^{2}\right) \sin (\eta / 2) \sum_{k=1}^{n} \gamma_{k}^{(n)} \cot \left(\frac{\eta-\theta_{k}^{(n)}}{2}\right)
$$

which is well defined for $\eta \in[0,2 \pi] \backslash\left\{\theta_{k}^{(n)}, 1 \leq k \leq n\right\}$, vanishes if and only if $\eta=\theta_{k}^{(n+1)}$ for some $k \in\{1, \ldots, n+1\}$, and one has the inequalities:

$$
0<\theta_{1}^{(n+1)}<\theta_{1}^{(n)}<\theta_{2}^{(n+1)}<\theta_{2}^{(n)}<\cdots<\theta_{n}^{(n+1)}<\theta_{n}^{(n)}<\theta_{n+1}^{(n+1)}<2 \pi .
$$

In other words, the eigenvalues of $u_{n+1}$ interlace between one and the eigenvalues of $u_{n}$.

Proof. The quantity $\gamma_{k}^{(n)}$ is well defined since $\mu_{1}^{(n)} \neq 0$, which implies that $1-\left|v_{n}\right|^{2}>0$. The equality $\sum_{k=1}^{n} \gamma_{k}^{(n)}=1$ comes from the fact that $x_{n+1}$ has norm 1. By Lemma 4.4, one has, for $z \notin\left\{\lambda_{1}^{(n)}, \ldots, \lambda_{n}^{(n)}\right\}$,

$$
P_{n+1}(z)=\frac{P_{n}(z)}{\bar{v}_{n}-1}\left[\left(z-v_{n}\right)\left(\bar{v}_{n}-1\right)-(z-1)\left(1-\left|v_{n}\right|^{2}\right) \sum_{k=1}^{n} \gamma_{k}^{(n)} \frac{\lambda_{k}^{(n)}}{z-\lambda_{k}^{(n)}}\right] .
$$

Using $\sum_{k=1}^{n} \gamma_{k}^{(n)}=1$, we obtain

$$
\begin{aligned}
P_{n+1}(z) & =\frac{P_{n}(z)}{\bar{v}_{n}-1}\left[\left(z-v_{n}\right)\left(\bar{v}_{n}-1\right)+\frac{(z-1)\left(1-\left|v_{n}\right|^{2}\right)}{2}-\frac{(z-1)\left(1-\left|v_{n}\right|^{2}\right)}{2} \sum_{k=1}^{n} \gamma_{k}^{(n)} \frac{z+\lambda_{k}^{(n)}}{z-\lambda_{k}^{(n)}}\right] \\
& =\frac{P_{n}(z)}{\bar{v}_{n}-1}\left[z \bar{v}_{n}+v_{n}-\frac{(1+z)\left(1+\left|v_{n}\right|^{2}\right)}{2}+\frac{(1-z)\left(1-\left|v_{n}\right|^{2}\right)}{2} \sum_{k=1}^{n} \gamma_{k}^{(n)} \frac{z+\lambda_{k}^{(n)}}{z-\lambda_{k}^{(n)}}\right] .
\end{aligned}
$$

Let us now fix arbitrarily a convention for the square root of complex numbers (e.g., for $z \neq 0$, we can define $\sqrt{z}$ as the square root of $z$ which has an argument in the interval $(-\pi / 2, \pi / 2])$. We can then define, for all $z \in \mathbb{C}^{*}$ :

$$
Q_{n}(z):=(\sqrt{z})^{-n} \sqrt{\overline{P_{n}(0)}} P_{n}(z) .
$$

Since

$$
P_{n+1}(0)=P_{n}(0) \frac{v_{n}-1}{\bar{v}_{n}-1},
$$


one has, for all $z \notin\left\{0, \lambda_{1}^{(n)}, \ldots, \lambda_{n}^{(n)}\right\}$,

$$
\begin{aligned}
Q_{n+1}(z)= & \pm \sqrt{\frac{\bar{v}_{n}-1}{z\left(v_{n}-1\right)} \frac{Q_{n}(z)}{\bar{v}_{n}-1}\left[z \bar{v}_{n}+v_{n}-\frac{(1+z)\left(1+\left|v_{n}\right|^{2}\right)}{2}\right.} \\
& \left.+\frac{(1-z)\left(1-\left|v_{n}\right|^{2}\right)}{2} \sum_{k=1}^{n} \gamma_{k}^{(n)} \frac{z+\lambda_{k}^{(n)}}{z-\lambda_{k}^{(n)}}\right] \\
= & \pm \frac{Q_{n}(z)}{\left|v_{n}-1\right|}\left[\sqrt{z} \bar{v}_{n}+\frac{v_{n}}{\sqrt{z}}-\frac{\left(\sqrt{z}+\frac{1}{\sqrt{z}}\right)\left(1+\left|v_{n}\right|^{2}\right)}{2}\right. \\
& \left.-\frac{\left(\sqrt{z}-\frac{1}{\sqrt{z}}\right)\left(1-\left|v_{n}\right|^{2}\right)}{2} \sum_{k=1}^{n} \gamma_{k}^{(n)} \frac{\sqrt{z} / \sqrt{\lambda_{k}^{(n)}}+\sqrt{\lambda_{k}^{(n)}} / \sqrt{z}}{\sqrt{z} / \sqrt{\lambda_{k}^{(n)}}-\sqrt{\lambda_{k}^{(n)}} / \sqrt{z}}\right] .
\end{aligned}
$$

Since $v_{n}=\rho_{n} \mathrm{e}^{\mathrm{i} \psi_{n}}$ for $\rho_{n} \in(0,1)$ and $\psi_{n} \in(-\pi, \pi]$, one has, for all $\eta \in[0,2 \pi] \backslash\left\{\theta_{k}^{(n)}, 0 \leq k \leq n\right\}$,

$$
\begin{aligned}
Q_{n+1}\left(\mathrm{e}^{\mathrm{i} \eta}\right)= & \pm \frac{Q_{n}\left(\mathrm{e}^{\mathrm{i} \eta}\right)}{\left|\rho_{n} \mathrm{e}^{\mathrm{i} \psi_{n}}-1\right|}\left[2 \rho_{n} \cos \left(\eta / 2-\psi_{n}\right)-\left(1+\rho_{n}^{2}\right) \cos (\eta / 2)\right. \\
& \left.-\left(1-\rho_{n}^{2}\right) \sin (\eta / 2) \sum_{k=1}^{n} \gamma_{k}^{(n)} \cot \left(\frac{\eta-\theta_{k}^{(n)}}{2}\right)\right] .
\end{aligned}
$$

Now, let us suppose that

$$
0<\theta_{1}^{(n)}<\theta_{2}^{(n)}<\cdots<\theta_{n}^{(n)}<2 \pi
$$

for some $n \geq 1$. The function $\Phi$, given in Lemma 4.5, is well defined and continuous on each of the intervals $\left[0, \theta_{1}^{(n)}\right),\left(\theta_{1}^{(n)}, \theta_{2}^{(n)}\right), \ldots,\left(\theta_{n-1}^{(n)}, \theta_{n}^{(n)}\right),\left(\theta_{n}^{(n)}, 2 \pi\right]$. Now, $\Phi(0)=1+$ $\rho_{n}^{2}-2 \rho_{n} \cos \left(\psi_{n}\right) \geq\left(1-\rho_{n}\right)^{2}>0$ (note that $\rho_{n}<1$, since $E_{0}$ holds), for all $k \in\{1, \ldots, n\}$, $\Phi(\eta)$ tends to $-\infty$ when $\eta$ tends to $\theta_{k}^{(n)}$ from below and to $+\infty$ when $\eta$ tends to $\theta_{k}^{(n)}$ from above (since $1-\rho_{n}^{2}, \sin (\eta / 2)$ and $\gamma_{k}^{(n)}$ are strictly positive), and $\Phi(2 \pi)=$ $-\Phi(0)<0$. One deduces that $\Phi(\eta)$ vanishes at least once on each of the intervals $\left(0, \theta_{1}^{(n)}\right),\left(\theta_{1}^{(n)}, \theta_{2}^{(n)}\right), \ldots,\left(\theta_{n-1}^{(n)}, \theta_{n}^{(n)}\right),\left(\theta_{n}^{(n)}, 2 \pi\right)$, in other words, there exists $\left(\tau_{k}\right)_{1 \leq k \leq n+1}$ such that

$$
0<\tau_{1}<\theta_{1}^{(n)}<\tau_{2}<\theta_{2}^{(n)}<\cdots<\tau_{n}<\theta_{n}^{(n)}<\tau_{n+1}<2 \pi
$$


and $\Phi\left(\tau_{k}\right)=0$ for all $k \in\{1, \ldots, n\}$. Now, by $(4.2), Q_{n+1}\left(\mathrm{e}^{\mathrm{i} \tau_{k}}\right)=0$, and then $P_{n+1}\left(\mathrm{e}^{\mathrm{i} \tau_{k}}\right)=0$, for all $k \in\{1,2, \ldots, n+1\}$. Hence, necessarily, $\tau_{k}=\theta_{k}^{(n+1)}$, which implies (4.1). In particular,

$$
0<\theta_{1}^{(n+1)}<\theta_{2}^{(n+1)}<\cdots<\theta_{n}^{(n+1)}<\theta_{n+1}^{(n+1)}<2 \pi
$$

and by induction, (4.3) and (4.1) hold for all $n \geq 1$.

In the following lemma, we define an event, which is almost surely satisfied under the Haar measure, and which is involved in a crucial way in our proof of Theorem 4.3.

Lemma 4.6. Let us suppose that $\left(u_{n}\right)_{n \geq 1}$ is a virtual isometry following the Haar measure, and let us take the notation above. Then, the event $E:=E_{0} \cap E_{1} \cap E_{2} \cap E_{3}$ holds almost surely, where

$$
\begin{aligned}
& E_{1}:=\left\{\exists n_{0} \geq 1, \forall n \geq n_{0}, \rho_{n} \leq n^{-0.4}\right\}, \\
& E_{2}:=\left\{\exists n_{0} \geq 1, \forall n \geq n_{0}, \forall k \in\{1, \ldots, n\}, \gamma_{k}^{(n)} \leq n^{-0.99}\right\}, \\
& E_{3}:=\left\{\exists n_{0} \geq 1, \forall n \geq n_{0}, \forall k \in \mathbb{Z}, n^{-1.7} \leq \theta_{k+1}^{(n)}-\theta_{k}^{(n)} \leq n^{-0.9}\right\} .
\end{aligned}
$$

Proof. It is easy to check that under the Haar measure on virtual isometries:

(1) the angle $\theta_{0}^{(1)}$ is uniform on $(-2 \pi, 0]$;

(2) for all $n \geq 1, \rho_{n}$ is the square root of a beta variable of parameters 1 and $n$;

(3) for all $n \geq 1, \psi_{n}$ is uniform on $(-\pi, \pi]$;

(4) for all $n \geq 1, k \in\{1,2, \ldots, n\}, \gamma_{k}^{(n)}=\left|\xi_{k}^{(n)}\right|^{2}$, where the vector $\left(\xi_{k}^{(n)}\right)_{1 \leq k \leq n}$ is uniform on the complex sphere of dimension $n$.

Moreover, the random variables $\theta_{0}^{(1)},\left(\rho_{n}\right)_{n \geq 1},\left(\psi_{n}\right)_{n \geq 1}$, and the random vectors $\left(\xi^{(n)}\right)_{n \geq 1}$ are independent. It is immediate to check that the condition $E_{0}$ holds almost surely. Hence, by Borel-Cantelli lemma, it is sufficient to check that for $n$ going to infinity:

$$
\begin{aligned}
& \mathbb{P}\left[\rho_{n}>n^{-0.4}\right]=O\left(n^{-1.1}\right), \\
& \mathbb{P}\left[\exists k \in\{1, \ldots, n\}, \gamma_{k}^{(n)}>n^{-0.99}\right]=O\left(n^{-1.1}\right), \\
& \mathbb{P}\left[\exists k \in \mathbb{Z}, \theta_{k+1}^{(n)}-\theta_{k}^{(n)}>n^{-0.9}\right]=O\left(n^{-1.1}\right), \\
& \mathbb{P}\left[\exists k \in \mathbb{Z}, \theta_{k+1}^{(n)}-\theta_{k}^{(n)}<n^{-1.7}\right]=O\left(n^{-1.1}\right) .
\end{aligned}
$$


Now, for all $n \geq 1, k \in\{1, \ldots, n+1\}, \rho_{n}^{2}$ and $\gamma_{k}^{(n+1)}$ have the same law as

$$
\frac{e_{1}}{e_{1}+e_{2}+\cdots+e_{n+1}}
$$

where $\left(e_{k}\right)_{1 \leq k \leq n+1}$ are independent standard exponential variables. Now, it is a classical result that the probabilities $\mathbb{P}\left[e_{1} \geq n^{0.001}\right]$ and $\mathbb{P}\left[e_{1}+\cdots+e_{n} \leq n / 2\right]$ decrease to zero faster than any negative power of $n$ when $n$ goes to infinity. Hence,

$$
\mathbb{P}\left[\frac{e_{1}}{e_{1}+e_{2}+\cdots+e_{n+1}}>n^{-0.99}\right]=O\left(n^{-28}\right),
$$

which easily implies (4.4) and (4.5). Now, the process of the eigenvalues of a random matrix following the Haar measure on $U(n)$ is a determinantal process, with kernel equal to $K^{(n)}(x)=\sin (n x / 2) /[2 \pi \sin (x / 2)]$, where $x$ denotes the difference between the two eigenangles which are considered. One deduces the following estimate for the two-point correlation function:

$$
\rho_{2}^{(n)}(u, v)=K^{(n)}(0)^{2}-K^{(n)}(u-v)^{2}=\mathrm{O}\left(n^{4}(u-v)^{2}\right) .
$$

The probability that there exist two eigenangles with distance smaller than or equal to $x$ is therefore dominated by

$$
\iint_{|u-v|<x} \rho_{2}^{(n)}(u, v) \mathrm{d} u \mathrm{~d} v=\mathrm{O}\left(n^{4} x^{3}\right)
$$

which implies (4.7). In order to prove (4.6), let us denote by $I$ a measurable subset of the interval $[0,2 \pi)$. The probability $\mathbb{P}_{I}^{(n)}$ that all the eigenangles of $u_{n}$ are in $I$ is (by the Andreiev-Heine identity, see, e.g., [14])

$$
\mathbb{P}_{I}^{(n)}=\operatorname{det}\left(M_{j, k}^{n, I}\right)_{1 \leq j, k, \leq n} \quad \text { where } M_{j, k}^{n, I}=\frac{1}{2 \pi} \int_{I} \mathrm{e}^{i(j-k) \theta} \mathrm{d} \theta
$$

Since for all $\theta \in[0,2 \pi)$, the matrix $\left(\mathrm{e}^{i(j-k) \theta}\right)_{1 \leq j, k \leq n}$ is hermitian and positive (its rank is 1 and its trace is $n), M^{n, I}$ and $M^{n, I^{c}}$ are also hermitian and positive. Moreover, $M^{n, I}+$ $M^{n, I^{c}}=\operatorname{Id}_{n}$, hence, the eigenvalues $\left(\tau_{j}\right)_{1 \leq j \leq n}$ of $M^{n, I^{c}}$ are in the interval [0, 1]. One deduces

$$
\mathbb{P}_{I}^{(n)}=\operatorname{det}\left(M^{n, I}\right)=\prod_{j=1}^{n}\left(1-\tau_{j}\right) \leq \exp \left(-\sum_{j=1}^{n} \tau_{j}\right) \leq \exp \left(-\operatorname{Tr}\left(M^{n, I^{c}}\right)\right)=\exp \left(-n \lambda\left(I^{c}\right) / 2 \pi\right)
$$


where $\lambda$ is the Lebesgue measure. Now, let us choose an integer $q \in\left[13 n^{0.9}, 14 n^{0.9}\right]$. For all $l \in\{0,1, \ldots q-1\}$,

$$
\mathbb{P}_{[2 \pi l / q, 2 \pi(l+1) / q)^{c}}^{(n)} \leq \mathrm{e}^{-n / q} \leq \mathrm{e}^{-n^{0.1} / 14},
$$

and then, with probability greater than or equal to $1-14 n^{0.9} \mathrm{e}^{-n^{0.1} / 14}, u_{n}$ has at least an eigenangle in each interval of the form $[2 \pi l / q, 2 \pi(l+1) / q)$. In this case, the maximal distance between two eigenangles is smaller than or equal to $4 \pi / q \leq 4 \pi n^{-0.9} / 13$, which implies (4.6).

Our interest in the event $E$ lies in the following result:

Lemma 4.7. Let $\left(u_{n}\right)_{n \geq 1}$ be a virtual isometry such that the event $E$ holds. Let us extend the notation $\gamma_{k}^{(n)}$ to all the values $k \in \mathbb{Z}$, in the unique way such that $\gamma_{k+n}^{(n)}=\gamma_{k}^{(n)}$. Then, for all $k \in \mathbb{Z}$, there exists $L \neq 0$ such that

$$
\theta_{k}^{(n)} \exp \left(\sum_{p=1}^{n-1} \gamma_{k}^{(p)}\right)=L+O\left(n^{-\varepsilon}\right)
$$

when $n$ goes to infinity, $\varepsilon>0$ being a universal constant.

Proof. Let us suppose $n \geq|k|+1$. Then, $\theta_{k}^{(n+1)} \in\left(\theta_{k-1}^{(n)}, \theta_{k}^{(n)}\right)$ for $k \geq 2, \theta_{k}^{(n+1)} \in\left(0, \theta_{1}^{(n)}\right)$ for $k=$ $1, \theta_{k}^{(n+1)} \in\left(\theta_{0}^{(n)}, 0\right)=\left(\theta_{n}^{(n)}-2 \pi, 0\right)$ for $k=0$, and $\theta_{k}^{(n+1)} \in\left(\theta_{k}^{(n)}, \theta_{k+1}^{(n)}\right)=\left(\theta_{n+k}^{(n)}-2 \pi, \theta_{n+k+1}^{(n)}-2 \pi\right)$ for $k \leq-1$. Moreover, by Lemma 4.5,

$$
\begin{aligned}
& \left(1+\rho_{n}^{2}\right) \cos \left(\theta_{k}^{(n+1)} / 2\right)-2 \rho_{n} \cos \left(\left(\theta_{k}^{(n+1)} / 2\right)-\psi_{n}\right) \\
& +\left(1-\rho_{n}^{2}\right) \sin \left(\theta_{k}^{(n+1)} / 2\right) \sum_{j=1}^{n} \gamma_{j}^{(n)} \cot \left(\frac{\theta_{k}^{(n+1)}-\theta_{j}^{(n)}}{2}\right)=0
\end{aligned}
$$

for $k \geq 1$, and

$$
\begin{aligned}
& \left(1+\rho_{n}^{2}\right) \cos \left(\theta_{n+k+1}^{(n+1)} / 2\right)-2 \rho_{n} \cos \left(\left(\theta_{n+k+1}^{(n+1)} / 2\right)-\psi_{n}\right) \\
& \quad+\left(1-\rho_{n}^{2}\right) \sin \left(\theta_{n+k+1}^{(n+1)} / 2\right) \sum_{j=1}^{n} \gamma_{j}^{(n)} \cot \left(\frac{\theta_{n+k+1}^{(n+1)}-\theta_{j}^{(n)}}{2}\right)=0,
\end{aligned}
$$


for $k \leq 0$, which also implies (4.8), since $\sin (x+\pi)=-\sin (x)$ and $\cos (x+\pi)=-\cos (x)$ for all $x \in \mathbb{R}$. Then, by the periodicity of the cotangent, one has

$$
\begin{aligned}
& \left(1+\rho_{n}^{2}\right) \cos \left(\theta_{k}^{(n+1)} / 2\right)-2 \rho_{n} \cos \left(\left(\theta_{k}^{(n+1)} / 2\right)-\psi_{n}\right) \\
& \quad+\left(1-\rho_{n}^{2}\right) \sin \left(\theta_{k}^{(n+1)} / 2\right) \sum_{j \in J} \gamma_{j}^{(n)} \cot \left(\frac{\theta_{k}^{(n+1)}-\theta_{j}^{(n)}}{2}\right)=0 .
\end{aligned}
$$

for any set $J$ consisting of $n$ consecutive integers. From now, we choose $J$ equal to the set of integers $j$ such that $\theta_{j}^{(n)} \in\left(\theta_{k}^{(n+1)}-\pi, \theta_{k}^{(n+1)}+\pi\right]$. Since the condition $E$ holds, one has the following estimates (for $k$ fixed and $n$ going to infinity):

$$
\begin{gathered}
1+\rho_{n}^{2}=1+O\left(n^{-0.8}\right), \\
\left|2 \rho_{n} \cos \left(\left(\theta_{k}^{(n+1)} / 2\right)-\psi_{n}\right)\right| \leq 2 \rho_{n}=O\left(n^{-0.4}\right), \\
\theta_{k}^{(n+1)}=O\left(n^{-0.9}\right), \\
\cos \left(\theta_{k}^{(n+1)} / 2\right)=1+O\left(n^{-1.8}\right), \\
\left(1+\rho_{n}^{2}\right) \cos \left(\theta_{k}^{(n+1)} / 2\right)-2 \rho_{n} \cos \left(\left(\theta_{k}^{(n+1)} / 2\right)-\psi_{n}\right)=1+O\left(n^{-0.4}\right),
\end{gathered}
$$

and then,

$$
\left(1-\rho_{n}^{2}\right) \sin \left(\theta_{k}^{(n+1)} / 2\right) \sum_{j \in J} \gamma_{j}^{(n)} \cot \left(\frac{\theta_{k}^{(n+1)}-\theta_{j}^{(n)}}{2}\right)=-1+O\left(n^{-0.4}\right) .
$$

Since $1-\rho_{n}^{2}=1+O\left(n^{-0.8}\right)$, and $\sin \left(\theta_{k}^{(n+1)} / 2\right)=\left(\theta_{k}^{(n+1)} / 2\right)\left(1+O\left(n^{-1.8}\right)\right)$, one deduces:

$$
\theta_{k}^{(n+1)} \sum_{j \in J} \gamma_{j}^{(n)} \cot \left(\frac{\theta_{k}^{(n+1)}-\theta_{j}^{(n)}}{2}\right)=-2+O\left(n^{-0.4}\right) .
$$

Now, since the function $x \rightarrow \cot (x)-1 / x$ is bounded on the interval $[-\pi / 2, \pi / 2]$,

$$
\theta_{k}^{(n+1)} \sum_{j \in J} \gamma_{j}^{(n)}\left[\cot \left(\frac{\theta_{k}^{(n+1)}-\theta_{j}^{(n)}}{2}\right)-\frac{2}{\theta_{k}^{(n+1)}-\theta_{j}^{(n)}}\right]
$$


is dominated by

$$
\left|\theta_{k}^{(n+1)}\right| \sum_{j \in J} \gamma_{j}^{(n)}=\left|\theta_{k}^{(n+1)}\right|=O\left(n^{-0.9}\right)
$$

which implies:

$$
\theta_{k}^{(n+1)} \sum_{j \in J} \frac{\gamma_{j}^{(n)}}{\theta_{k}^{(n+1)}-\theta_{j}^{(n)}}=-1+O\left(n^{-0.4}\right)
$$

Note that

$$
\theta_{k+n+1}^{(n)}=\theta_{k+1}^{(n)}+2 \pi \geq \theta_{k}^{(n+1)}+2 \pi
$$

and

$$
\theta_{k-n-1}^{(n)}=\theta_{k-1}^{(n)}-2 \pi \leq \theta_{k}^{(n+1)}-2 \pi,
$$

hence, all the elements of $J$ are included in the interval $[k-n, k+n]$. Moreover, for $n$ large enough and for all integers $p \geq 1$ :

$$
\theta_{k+p+1}^{(n)}-\theta_{k}^{(n+1)} \geq \theta_{k+p+1}^{(n)}-\theta_{k+1}^{(n)} \geq p n^{-1.7}
$$

and

$$
\theta_{k}^{(n+1)}-\theta_{k-p-1}^{(n)} \geq \theta_{k-1}^{(n)}-\theta_{k-p-1}^{(n)} \geq p n^{-1.7}
$$

One deduces that, for $n$ large enough:

$$
\begin{aligned}
\left|\theta_{k}^{(n+1)} \sum_{j \in J,|j-k|>1} \frac{\gamma_{j}^{(n)}}{\theta_{k}^{(n+1)}-\theta_{j}^{(n)}}\right| & \leq 2\left|\theta_{k}^{(n+1)}\right|\left(\sup _{1 \leq j \leq n} \gamma_{j}^{(n)}\right)\left(\sum_{p=1}^{n} \frac{1}{p n^{-1.7}}\right) \\
& =O\left(n^{-0.9}\right) . O\left(n^{-0.99}\right) . O\left(n^{1.7} \log n\right)=O\left(n^{-0.1}\right) .
\end{aligned}
$$

On the other hand,

$$
0 \leq \theta_{k}^{(n+1)}-\theta_{k-1}^{(n)} \leq \theta_{k+1}^{(n)}-\theta_{k-1}^{(n)}=O\left(n^{-0.9}\right)
$$

and

$$
0 \leq \theta_{k+1}^{(n)}-\theta_{k}^{(n+1)} \leq \theta_{k+1}^{(n)}-\theta_{k-1}^{(n)}=O\left(n^{-0.9}\right)
$$

hence, $k-1, k, k+1 \in J$ for $n$ large enough. One deduces:

$$
\theta_{k}^{(n+1)}\left(\frac{\gamma_{k-1}^{(n)}}{\theta_{k}^{(n+1)}-\theta_{k-1}^{(n)}}+\frac{\gamma_{k}^{(n)}}{\theta_{k}^{(n+1)}-\theta_{k}^{(n)}}+\frac{\gamma_{k+1}^{(n)}}{\theta_{k}^{(n+1)}-\theta_{k+1}^{(n)}}\right)=-1+O\left(n^{-0.1}\right)
$$


Therefore, for $n$ large enough, there exists $j \in\{k-1, k, k+1\}$ such that

$$
\frac{\theta_{k}^{(n+1)} \gamma_{j}^{(n)}}{\theta_{k}^{(n+1)}-\theta_{j}^{(n)}}<-\frac{1}{4}
$$

One deduces that $\theta_{k}^{(n+1)}-\theta_{j}^{(n)}=O\left(n^{-1.89}\right)$, since $\theta_{k}^{(n+1)} \gamma_{j}^{(n)}=O\left(n^{-1.89}\right)$. Now, let us suppose that $j=k+1$. In this case, $\theta_{k}^{(n+1)}-\theta_{j}^{(n)}$ is negative, and then $\theta_{k}^{(n+1)}$ should be positive, and $k$ should be strictly positive. Then, $\theta_{k}^{(n)}-\theta_{k}^{(n+1)}>0$ and

$$
\theta_{j}^{(n)}-\theta_{k}^{(n+1)} \geq\left(\theta_{k+1}^{(n)}-\theta_{k}^{(n)}\right)+\left(\theta_{k}^{(n)}-\theta_{k}^{(n+1)}\right) \geq n^{-1.7},
$$

which is a contradiction for $n$ large enough. Similarly, if $j=k-1, \theta_{k}^{(n+1)}-\theta_{j}^{(n)}$ is positive, $\theta_{k}^{(n+1)}$ is negative, $k \leq 0, \theta_{k}^{(n)}-\theta_{k}^{(n+1)}<0$ and $\theta_{k}^{(n+1)}-\theta_{j}^{(n)} \geq n^{-1.7}$, which is again a contradiction. Therefore, $j=k$ for $n$ large enough and $\theta_{k}^{(n+1)}-\theta_{k}^{(n)}=O\left(n^{-1.89}\right)$. One deduces that for $j \in\{k-1, k+1\}$,

$$
\left|\theta_{k}^{(n+1)}-\theta_{j}^{(n)}\right| \geq\left|\theta_{k}^{(n)}-\theta_{j}^{(n)}\right|-\left|\theta_{k}^{(n+1)}-\theta_{k}^{(n)}\right| \geq n^{-1.7}-O\left(n^{-1.89}\right) \geq n^{-1.7} / 2
$$

if $n$ is large enough, and then

$$
\frac{\theta_{k}^{(n+1)} \gamma_{j}^{(n)}}{\theta_{k}^{(n+1)}-\theta_{j}^{(n)}}=O\left(n^{-0.1}\right)
$$

Consequently, one has

$$
\frac{\theta_{k}^{(n+1)} \gamma_{k}^{(n)}}{\theta_{k}^{(n+1)}-\theta_{k}^{(n)}}=-1+O\left(n^{-0.1}\right)
$$

and since $\theta_{k}^{(n+1)} \neq 0$,

$$
\frac{\gamma_{k}^{(n)}}{1-\left(\theta_{k}^{(n)} / \theta_{k}^{(n+1)}\right)}=-1+O\left(n^{-0.1}\right)
$$

which implies

$$
\gamma_{k}^{(n)}=\left(\frac{\theta_{k}^{(n)}}{\theta_{k}^{(n+1)}}-1\right)\left(1+O\left(n^{-0.1}\right)\right)
$$


In particular, $\left(\theta_{k}^{(n)} / \theta_{k}^{(n+1)}\right)-1$ is equivalent to $\gamma_{k}^{(n)}$, and then, dominated by $n^{-0.99}$. One deduces:

$$
\begin{aligned}
\gamma_{k}^{(n)} & =\left(\frac{\theta_{k}^{(n)}}{\theta_{k}^{(n+1)}}-1\right)+O\left(n^{-0.99}\right) \cdot O\left(n^{-0.1}\right), \\
\frac{\theta_{k}^{(n)}}{\theta_{k}^{(n+1)}} & =1+\gamma_{k}^{(n)}+O\left(n^{-1.09}\right),
\end{aligned}
$$

and then

$$
\log \left(\frac{\theta_{k}^{(n)}}{\theta_{k}^{(n+1)}}\right)=\gamma_{k}^{(n)}+O\left(n^{-1.09}\right)
$$

Now, if one sets

$$
L_{n}:=\log \left(\left|\theta_{k}^{(n)}\right|\right)+\sum_{p=1}^{n-1} \gamma_{k}^{(p)}
$$

then $L_{n+1}-L_{n}=O\left(n^{-1.09}\right)$. One deduces that $\left(L_{n}\right)_{n \geq 1}$ converges to a limit $L_{\infty}$ when $n$ goes to infinity, with $L_{\infty}-L_{n}=O\left(n^{-0.09}\right)$. Taking the exponential ends the proof of Lemma 4.7, for $\varepsilon=0.09$.

We now have all the ingredients involved in the proof of Theorem 4.3. Indeed, by Lemma 4.7, there exists a random variable $L \neq 0$ such that almost surely, for $n$ going to infinity,

$$
\theta_{k}^{(n)} \exp \left(\sum_{p=1}^{n-1} \gamma_{k}^{p}\right)=n \theta_{k}^{(n)} \exp \left(-\log n+\sum_{p=1}^{n-1} \frac{1}{p}\right) \exp \left(M_{n}\right)=L+O\left(n^{-\varepsilon}\right)
$$

where

$$
M_{n}:=\sum_{p=1}^{n-1}\left(\gamma_{k}^{(p)}-\frac{1}{p}\right)
$$

since the event $E$ holds almost surely. Now, the variables $\left(\gamma_{k}^{(n)}\right)_{n \geq 1}$ are independent, with expectation $1 / n$, and then the process $\left(M_{n}\right)_{n \geq 1}$ is a martingale with respect to the 
filtration generated by $\left(\gamma_{k}^{(n)}\right)_{n \geq 1}$. Moreover, with the notation above,

$$
\begin{aligned}
\mathbb{E}\left[\left(\gamma_{k}^{(n)}-\frac{1}{n}\right)^{2}\right] & =\mathbb{E}\left[\left(\frac{e_{1}}{e_{1}+e_{2}+\cdots+e_{n}}-\frac{1}{n}\right)^{2}\right] \\
& =\mathbb{E}\left[\left(\frac{(n-1) e_{1}-e_{2}-e_{3}-\cdots-e_{n}}{n\left(e_{1}+e_{2}+\cdots+e_{n}\right)}\right)^{2}\right] \\
& \leq \mathbb{P}\left[e_{1}+\cdots+e_{n} \leq n / 2\right]+\frac{4}{n^{4}} \mathbb{E}\left[\left((n-1) e_{1}-e_{2}-e_{3}-\cdots-e_{n}\right)^{2}\right] \\
& \leq O\left(n^{-28}\right)+\frac{4}{n^{4}}\left[\operatorname{Var}\left((n-1) e_{1}\right)+\operatorname{Var}\left(e_{2}\right)+\cdots+\operatorname{Var}\left(e_{n}\right)\right] \\
& =O\left(n^{-28}\right)+\frac{4 n(n-1)}{n^{4}}=O\left(1 / n^{2}\right) .
\end{aligned}
$$

Hence, the martingale $\left(M_{n}\right)_{n \geq 1}$ is bounded in $L^{2}$, and then, converges almost surely (and in $L^{2}$ ) to a limit random variable $M_{\infty}$. More precisely, for $n \geq 1$,

$$
\mathbb{E}\left[\left(M_{\infty}-M_{n}\right)^{2}\right]=\sum_{m=n}^{\infty} \mathbb{E}\left[\left(\gamma_{k}^{(m)}-\frac{1}{m}\right)^{2}\right]=O(1 / n),
$$

and by applying Doob's inequality to the martingale $\left(M_{2^{q}+m}\right)_{m \geq 0}$, for $q \geq 0$,

$$
\begin{aligned}
\mathbb{E}\left[\sup _{n \geq 2^{q}}\left(M_{\infty}-M_{n}\right)^{2}\right] & \leq 2\left(\mathbb{E}\left[\left(M_{\infty}-M_{2^{q}}\right)^{2}\right]+\mathbb{E}\left[\sup _{n \geq 2^{q}}\left(M_{n}-M_{2^{q}}\right)^{2}\right]\right) \\
& \leq 10 \mathbb{E}\left[\left(M_{\infty}-M_{2^{q}}\right)^{2}\right]=O\left(2^{-q}\right),
\end{aligned}
$$

when $q$ goes to infinity. Hence,

$$
\mathbb{P}\left[\sup _{n \geq 2^{q}}\left|M_{\infty}-M_{n}\right| \geq 2^{-q / 4}\right] \leq 2^{q / 2} \mathbb{E}\left[\sup _{n \geq 2^{q}}\left(M_{\infty}-M_{n}\right)^{2}\right]=O\left(2^{-q / 2}\right),
$$

and by Borel-Cantelli's lemma,

$$
\mathbb{P}\left[\exists q_{0} \geq 1, \forall q \geq q_{0}, \quad \forall n \geq 2^{q},\left|M_{\infty}-M_{n}\right| \geq 2^{-q / 4}\right]=1
$$

Therefore, almost surely,

$$
\left|M_{\infty}-M_{n}\right|=O\left(n^{-1 / 4}\right)
$$


when $n$ goes to infinity. Now, by (4.9), one has almost surely:

$$
\begin{aligned}
n \theta_{k}^{(n)} / 2 \pi & =\frac{1}{2 \pi}\left(L+O\left(n^{-\varepsilon}\right)\right) \exp \left(-M_{n}\right) \exp \left(\log n-\sum_{p=1}^{n-1} \frac{1}{p}\right) \\
& =\frac{1}{2 \pi}\left(L+O\left(n^{-\varepsilon}\right)\right) \exp \left(-M_{\infty}+O\left(n^{-1 / 4}\right)\right) \exp \left(-\gamma+O\left(n^{-1}\right)\right) \\
& =x_{k}+O\left(n^{-\varepsilon}\right),
\end{aligned}
$$

where $\gamma$ is Euler constant and

$$
X_{k}=\frac{L}{2 \pi \mathrm{e}^{\gamma+M_{\infty}}}
$$

if one assumes $\varepsilon \leq \frac{1}{4}$. Moreover, since one knows that the point process $\left(n \theta_{k}^{(n)} / 2 \pi\right)_{k \in \mathbb{Z}}$ converges weakly to a determinantal process with sine kernel, the limit point process $\left(x_{k}\right)_{k \in \mathbb{Z}}$ is also necessarily a determinantal process with sine kernel.

Remark. This probabilistic proof of almost sure convergence to a sine point process can be extended to Hua-Pickrell measures. This requires showing analogs of Equations (4.4)-(4.7), hence a precise analysis on the hypergeometric kernel, which is not the purpose of this article. Theorem 4.3 is the exact equivalent to Corollary 4.2, for unitary matrices. The link between Theorem 4.1 and Corollary 4.2 shows immediately that the behavior of the large cycles of random permutations is strongly related to the behavior of the corresponding eigenvalues which are close to 1. Similarly, the behavior of the small cycles of a permutation is directly related to the traces of the small powers of the corresponding matrix. Now, if for $n, p \geq 1, Y_{p}^{(n)}$ denotes the number of $p$-cycles of a random permutation on $\mathcal{S}_{n}$ which follows the Ewens measure of parameter $\theta>0$, then for all $p_{0} \geq 1$, the joint distribution of $\left(Y_{p}^{(n)}\right)_{1 \leq p \leq p_{0}}$ tends to the distribution of $\left(Y_{p}\right)_{1 \leq p \leq p_{0}}$, where $\left(Y_{p}\right)_{p \geq 1}$ is a sequence of independent Poisson random variables such that $\mathbb{E}\left[Y_{p}\right]=\theta / p$. This result can easily be translated to a result of weak convergence for the finitedimensional marginales of the sequence $\left(\operatorname{Tr}\left(u_{n}^{p}\right)\right)_{p \geq 1}$, where $u_{n}$ is a random permutation matrix of order $n$, which follows the Ewens measure of parameter $\theta$. For general unitary matrices, one has a similar result on the traces: if $u_{n}$ is a random unitary matrix of order $n$ which follows the Haar measure, then the finite-dimensional marginales of $\left(\operatorname{Tr}\left(u_{n}^{p}\right)\right)_{p \geq 1}$ converge in law to sequences of i.i.d. complex gaussian random variables [3]. 


\section{Funding}

This research was supported in part by Schweizerischer Nationalfonds Projekte Nr. 200020131965 (to A.N.).

\section{References}

[1] Borodin, A. and G. Olshanski. "Infinite random matrices and ergodic measures." Communications in Mathematical Physics 223, no. 1 (2001): 87-123.

[2] Bourgade, P., A. Nikeghbali, and A. Rouault. "Ewens measures on compact groups and hypergeometric kernels." Séminaire de probabilités 43, LNM 2006 (2011): 351-377.

[3] Diaconis, P. and M. Shahshahani. "On the eigenvalues of random matrices." Journal of Applied Probability 31A (1994): 49-62. Studies in applied probability.

[4] Hua, L. K. Harmonic Analysis of Functions of Several Complex Variables in the Classical Domains. Translated from the Russian by Leo Ebner and Adam Korányi. Providence, RI: American Mathematical Society, 1963.

[5] Kerov, S.-V., G.-I. Olshanski, and A.-M. Vershik. "Harmonic analysis on the infinite symmetric group." Comptes Rendus de l'Académie des sciences de Paris 316, no. 8 (1993): 773-8.

[6] Kingman, J.-F.-C. "Random discrete distribution." Journal of the Royal Statistical Society Series B 37, no. 1 (1975): 1-22.

[7] Kingman, J.-F.-C. "Random partitions in population genetics." Proceedings of The Royal Society of London. Series A 361, no. 1704 (1978): 1-20.

[8] Kingman, J.-F.-C. "The representation of partition structures." J. Journal of the London Mathematical Society. Second Series 18, no. 2 (1978): 374-80.

[9] Neretin, Y.-A. "Hua type integrals over unitary groups and over projective limits of unitary groups." Duke Mathematical Journal 114, no. 2 (2002): 239-66.

[10] Olshanski, G. and A. Vershik, "Ergodic unitarily invariant measures on the space of infinite Hermitian matrices." Transactions of the American Mathematical Society. Second Series 175 (1996): 137-75.

[11] Pickrell, D. "Measures on infinite-dimensional Grassmann manifolds." Journal of Functional Analysis 70, no. 2 (1987): 323-356.

[12] Pickrell, D. "Mackey analysis of infinite classical motion groups." Pacific Journal of Mathematics 150, no. 1 (1991): 139-166.

[13] Pitman, J. Combinatorial Stochastic Processes. Lecture Notes in Mathematics 1875. Berlin: Springer, 2006.

[14] Soshnikov, A. "Determinantal random point fields." Russian Mathematical Surveys 55, no. 5 (2000): 923-75.

[15] Tsilevich, N.-V. "Distribution of cycle lengths of infinite permutations." Journal of Mathematical Sciences (New York) 87, no. 6 (1997): 4072-81.

[16] Tsilevich, N.-V. "Stationary measures on the space of virtual permutations for an action of the infinite symmetric group." Preprint St. Petersburg Dept. Steklov Math. Inst., PDMI13/1998, in press. 